\title{
Interval Tuning in the Primate Medial Premotor Cortex as a General Timing Mechanism
}

\author{
Hugo Merchant, Oswaldo Pérez, Wilbert Zarco, and Jorge Gámez \\ Instituto de Neurobiología, Universidad Nacional Autónoma de México, Campus Juriquilla, Quéretaro, México 76230
}

The precise quantification of time during motor performance is critical for many complex behaviors, including musical execution, speech articulation, and sports; however, its neural mechanisms are primarily unknown. We found that neurons in the medial premotor cortex (MPC) of behaving monkeys are tuned to the duration of produced intervals during rhythmic tapping tasks. Interval-tuned neurons showed similar preferred intervals across tapping behaviors that varied in the number of produced intervals and the modality used to drive temporal processing. In addition, we found that the same population of neurons is able to multiplex the ordinal structure of a sequence of rhythmic movements and a wide range of durations in the range of hundreds of milliseconds. Our results also revealed a possible gain mechanism for encoding the total number of intervals in a sequence of temporalized movements, where intervaltuned cells show a multiplicative effect of their activity for longer sequences of intervals. These data suggest that MPC is part of a core timing network that uses interval tuning as a signal to represent temporal processing in a variety of behavioral contexts where time is explicitly quantified.

\section{Introduction}

Temporal processing in the hundreds of milliseconds is a fundamental component of many complex behaviors, such as speech perception and articulation (Diehl et al., 2004), the execution and appreciation of music (Janata and Grafton, 2003), and sports performance (Merchant and Georgopoulos, 2006). Human subjects have the ability to quantify single or multiple intervals, defined by different sensory modalities, in a variety of perceptual or motor activities. Thus, a central question in timing research is whether a single neural mechanism is used for the measurement of time across different behaviors or if, on the contrary, multiple encoding strategies are used by the brain depending on the behavioral context in which time is processed (Merchant et al., 2013). Modeling studies have suggested that the representation of time is ubiquitous, arising from the intrinsic dynamics of nondedicated neural mechanisms (Karmarkar and Buonomano, 2007; Buonomano and Laje, 2010). In contrast, functional imaging studies using perceptual or motor tasks, with single or multiple time intervals and different sensory modalities used to define intervals, consistently found that structures such as the neostriatum and the supplementary motor areas, which are part of the cortico-basal ganglia-thalamocortical circuit (CBGT), are activated regardless of the nontiming factors involved in the task (Macar et al., 2006;

\footnotetext{
Received Nov. 28, 2012; revised April 8, 2013; accepted April 12, 2013

Author contributions: H.M. designed research; H.M. and W.Z. performed research; 0.P. contributed unpublished reagents/analytic tools; H.M., O.P., W.Z., and J.G. analyzed data; H.M. wrote the paper.

This work was supported by CONACYT Grant 151223 and PAPIIT Grant IN200511. We thank Bruno Averbeck, David Crowe, Victor de Lafuente, and Ranulfo Romo for fruitful comments on this manuscript. We also thank Luis Prado, Raul Paulín, and Juan Jose Ortiz for technical assistance.

Correspondence should be addressed to Dr. Hugo Merchant at the above address. E-mail: hugomerchant@unam.mx, merch006@umn.edu.

D01:10.1523/JNEUROSC1.5513-12.2013

Copyright $\odot 2013$ the authors $\quad 0270-6474 / 13 / 339082-15 \$ 15.00 / 0$
}

Harrington et al., 2010). Furthermore, in accordance with recent neuroimaging studies (Bueti et al., 2008), psychophysical research using multiple timing tasks supports neither a common nor multiple context-dependent timing mechanisms (Merchant et al., 2008a,b). Therefore, a parsimonious hypothesis is that timing depends on the interplay between multipurpose timing structures such as CBGT and areas that are selectively engaged depending on the specific requirements of a task (Coull et al., 2011; Merchant et al., 2013).

Psychophysical studies on learning and generalization of time intervals give support to the notion that neurons in the timing circuit are tuned to specific intervals but can be activated in a modality- and context-independent fashion (Nagarajan et al., 1998; Meegan et al., 2000; Bartolo and Merchant, 2009). Therefore, the change in discharge rate as a function of duration could be a neural code used by the CBGT to represent the passage of time (Matell et al., 2003a), as it has been shown for spatial and numerical variables (Buhusi and Meck, 2005; Nieder et al., 2006). Nevertheless, it remains unknown whether cells in the core timing circuit are tuned to intervals and whether the CBGT encodes temporal information similarly across behavioral contexts, number of temporalized intervals, and sensory modalities. Consequently, in the present study we examined the response properties of medial premotor cortex (MPC) cells during the execution of two rhythmic tapping tasks where the intervals were defined by auditory or visual cues. The results showed that MPC cells are tuned to the duration of intervals during rhythmic tapping. Interestingly, the interval-tuned neurons showed similar preferred intervals (PIs) across modalities during single interval reproduction and synchronization-continuation tasks. These data suggest that MPC is part of a core timing circuit that uses interval tuning as a signal to represent temporal information in a variety of timing behaviors. 
A
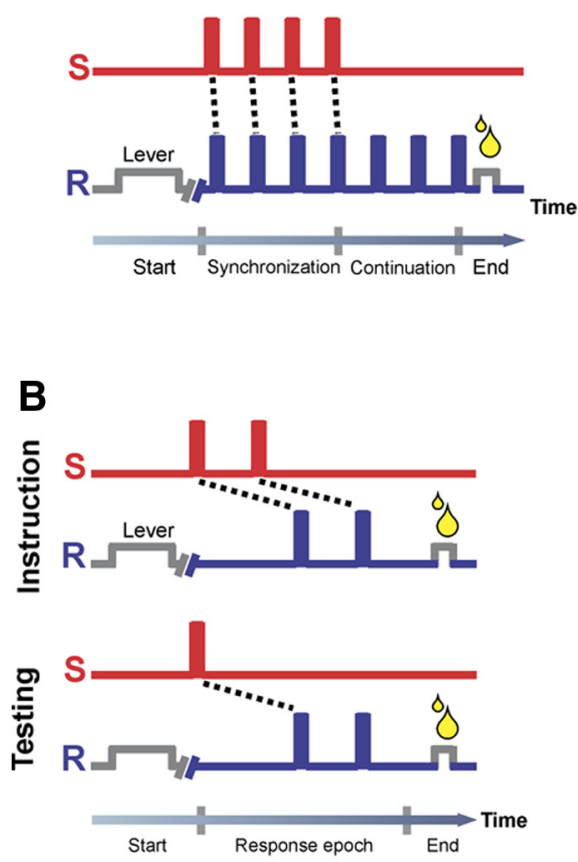

C

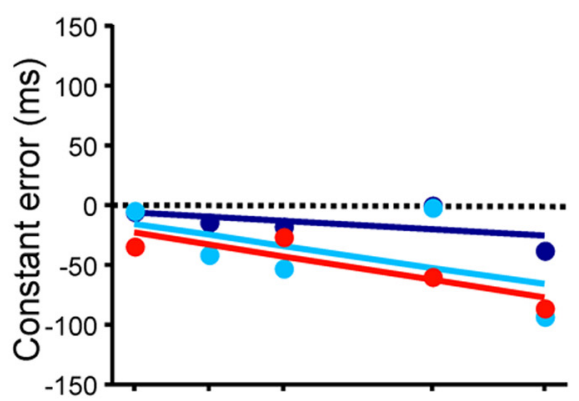

E

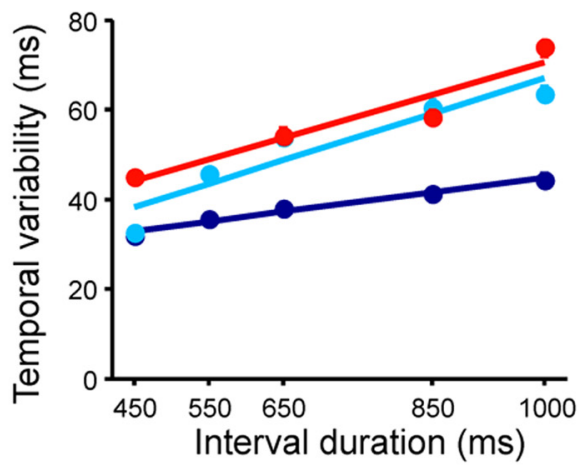

G

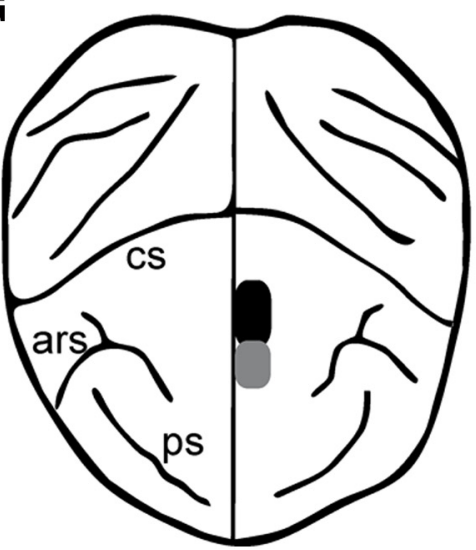

D

Visual

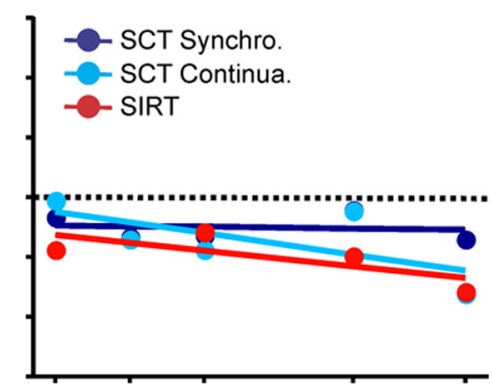

F

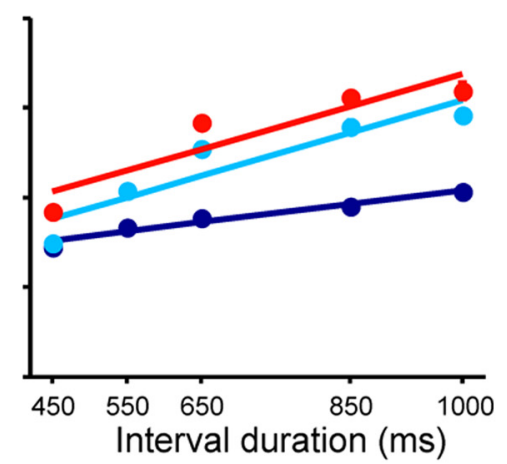

Figure 1. Tapping tasks, behavioral performance, and neural recordings. $A$, SCT. Monkeys were required to push a button (R, blue line) each time stimuli with a constant interstimulus interval (S, red line) were presented, which resulted in a stimulus-movement cycle. After four consecutive synchronized movements, the stimuli stopped, and the monkeys continued tapping with a similar interval for three additional intervals. The target intervals, defined by brief auditory or visual stimuli, were 450, 550,650, 850, and $1000 \mathrm{~ms}$ and were chosen pseudo-randomly within a repetition. $B$, SIRT. For each interval, there were training and testing periods. In the training period, the target interval (450,650, 850, or $1000 \mathrm{~ms})$ was presented at the beginning of the trial. Then, the animal tapped twice on the button to produce the same interval. This was repeated for five training trials, after which the monkey entered the testing period, where he produced another 10 single intervals, each in response to the presentation of a single stimulus. $\boldsymbol{C}, \boldsymbol{D}$, Constant error (produced-target interval) during the performance of both tasks using auditory $(\boldsymbol{C})$ and visual $(\boldsymbol{D})$ interval markers. Monkeys slightly underestimated the intervals during the synchronization (Synchro; blue) and continuation (Continua; cyan) phases of SCT, as well as during the SIRT (red). The SEM is smaller than the dot diameter. $\boldsymbol{E}, \boldsymbol{F}$, Temporal variability increased as a function of target interval in the auditory $(\boldsymbol{E})$ and visual $(\boldsymbol{F})$ interval marker conditions, during both phases of SCT and during SIRT. $\boldsymbol{G}$, Location of the recording area in SMA (black oval) and pre-SMA (gray oval) in a top view of the monkey brain. cs, (entral sulcus; ars, arcuate sulcus; ps, principal sulcus.

\section{Materials and Methods}

General

All the animal care, housing, and experimental procedures were approved by the National University of Mexico Institutional Animal Care and Use Committee and conformed to the principles outlined in the Guide for the Care and Use of Laboratory Animals (NIH, publication number 85-23, revised 1985). The two monkeys (Macaca mulatta, both males, $5-7 \mathrm{~kg}$ body weight) were monitored daily by the researchers and the animal care staff, and every second day by the veterinarian, to check the conditions of health and welfare. To ameliorate their condition of life, we routinely introduced in the home cage $\left(1.3 \mathrm{~m}^{3}\right)$ environment toys (often containing items of food that they liked) to promote their exploratory behavior. The researcher that tested the animals spent half an hour interacting with the monkeys directly, giving, for example, new objects to manipulate.

Synchronization-continuation task. The synchronization-continuation task (SCT) used in this study has been described previously (Zarco et al., 2009; Merchant et al., 2011). Briefly, the monkeys were required to push a button each time stimuli with a constant interstimulus interval were presented, which resulted in a stimulus-movement cycle (Fig. 1A). After four consecutive synchronized movements, the stimuli were eliminated, and the monkeys continued tapping with the same interval for three additional intervals. To avoid a preference toward short intervals, the reward amount was adjusted as a function of target durations, with longer durations giving greater amounts of juice, as described previously (Zarco et al., 2009). Trials were separated by a variable intertrial interval 
(1.2-4 s). The target intervals, defined by brief auditory $(33 \mathrm{~ms}, 2000 \mathrm{~Hz}, 65 \mathrm{~dB})$ or visual (4 cm side green square, $33 \mathrm{~ms}$ ) stimuli, were 450, 550, 650, 850, and $1000 \mathrm{~ms}$ and were chosen pseudo-randomly within a repetition. Five repetitions were collected for each target interval and modality.

Single interval reproduction task. For each interval, there were training and testing periods (Fig. $1 B$ ). In the training, the target interval $(450,650,850$, or $1000 \mathrm{~ms}$ presented in blocks of trials) was presented at the beginning of the trial. Then, the monkey tapped twice on the push-button to reproduce this interval. After five training trials, the animal entered the testing period, where it reproduced another 10 single intervals with the same duration, each in response to a single stimulus that acted as a go signal. The duration of each interval was associated with a particular stimulus feature so that during the testing period, the go signal was a stimulus that had been linked to the production of a specific interval during the training period. Thus, a $4400 \mathrm{~Hz}$ tone or a blue square was associated with the reproduction of $450 \mathrm{~ms}$, a $3000 \mathrm{~Hz}$ tone or green square with $650 \mathrm{~ms}$, a $1000 \mathrm{~Hz}$ tone or cyan square with $850 \mathrm{~ms}$, and a $650 \mathrm{~Hz}$ tone or yellow square with $1000 \mathrm{~ms}$. The target intervals were chosen pseudo-randomly between blocks. A total of 60 trials ( 40 for the testing period) were collected. Monkeys were rewarded following the same rules described in the SCT (Zarco et al., 2009). Throughout the experiment, trials were separated by a variable $1.2-4 \mathrm{~s}$ intertrial interval.

Procedure. A block of tasks consisted in the random order execution of the four task combinations: SCT and single interval reproduction task (SIRT), with visual and auditory interval marker conditions. During each day of recordings, the animals performed between two and five blocks of tasks. The same monkeys used by Merchant et al. (2011) were used in the present study. It is important to emphasize that we used the same durations in the SCT and SIRT. These intervals correspond to the hundreds of milliseconds range, as in previous time perception experiments (Leon and Shadlen, 2003; Harrington et al., 2010), but not as in previous time production experiments where intervals in the seconds range were used (Rakitin et al., 1998; Mita et al., 2009). Therefore, in the present study, we were able to investigate the neural underpinnings of temporal processing in the hundreds of milliseconds range during the execution of rhythmic sequences that share many components of musical execution, as well as during the production of single intervals.

Neural recordings. The extracellular activity of single neurons in the medial premotor areas was recorded using a system with seven independently movable microelectrodes (1-3 M $\Omega$; Uwe Thomas Recording) (Merchant et al., 2004). All the isolated neurons were recorded regardless of their activity during the task, and the recording sites changed from session to session. At each site, raw extracellular membrane potentials were sampled at $40 \mathrm{kHz}$. Single-unit activity was extracted from these records using the Plexon off-line sorter (Plexon). Structural magnetic resonance imaging was used to localize the recording sites (Merchant et al., 2011). Capillary tubes filled with vitamin-E oil were placed in the internal phase and on top of the $1.5 \mathrm{~cm}$ circular recording chambers and were used as markers to determine the anteroposterior and mediolateral location of the electrode penetrations (Fig. 1G).

\section{Data analysis}

General. Subroutines written in Matlab (version 7.6.0.324; Mathworks) and the SPSS statistical package (version 12, 2003; SPSS) were used for the statistical analyses. The level of statistical significance to reject the null hypothesis was $\alpha=0.05$. An initial ANOVA was performed for each neuron to identify cells whose activity changed significantly during the recording session. Of a total of 1570 cells recorded in the MPC in both monkeys (1267 in monkey 1 and 303 in monkey 2), 993 did not show a statistically significant effect of recording time during the key hold control period and were analyzed further. In this study, we do not address functional differences between SMA versus pre-SMA, since similar neural signals were observed in both areas. All these neurons were recorded for 5 repetitions during the SCT and for 15 repetitions during the SIRT; however, for the latter only the 10 trials corresponding to the testing phase were analyzed in the present study.

Timing behavior. Two parameters were evaluated as a measure of subject performance: the variance and the constant error. The mean and SD of each intertap interval for each monkey were used to compute the constant error and the variance, respectively. This implies that for the SCT, the variance corresponded to a general measure of within- and between-trial variability without averaging across trials in the synchronization and continuation phases. In accordance, in the SIRT the variance corresponded to the between-trial variability, since only one interval per trial was produced. The constant error was defined as the difference between the mean of the produced intervals minus the target interval. In a previous study, we compared the performance of human subjects and three monkeys during the two tasks using auditory or visual interval markers (Zarco et al., 2009). The results showed that the time subestimation and the increase in temporal variability as a function of the interval were similar to the data shown in Figure $1 C-F$ for the two monkeys performing the tasks during the electrophysiological recording sessions of the present paper.

Computing the discharge rate. The duration of target intervals varied systematically in the SCT and SIRT. Therefore, we could not use a fixed temporal window to compute the discharge rate of the cells, since it could artificially produce a bias in discharge rate toward shorter intervals. Instead, we used the Poisson train analysis (Hanes et al., 1995) to identify the periods of cell activation within each produced interval. This analysis determines how improbable it is that the number of action potentials within a specific condition (i.e., target interval and ordinal sequence) was a chance occurrence. For this purpose, the actual number of spikes within a time window was compared with the number of spikes predicted by the Poisson distribution derived from the mean discharge rate during the entire recording of the cell. The measure of improbability was the surprise index $(S I)$ defined as follows:

$$
S I=-\log P,
$$

where $P$ was defined by the following Poisson equation:

$$
P=e^{-r T} \sum_{i=n}^{\infty} \frac{(r T)^{i}}{i !},
$$

where $P$ is the probability that, given the average discharge rate $r$, a spike train of a produced interval $T$ contains $n$ or more spikes in a trial. Thus, a large SI indicates a low probability that a specific elevation in activity was a chance occurrence. This analysis assumes that an activation period is statistically different from the average discharge rate $r$, considering that the firing of the cell is following a nonhomogenous Poisson process (see also Perez et al., 2013).

The spike train analysis was applied for all trials of each produced interval in the SCT ( 30 produced intervals, 5 duration $\times 6$ intervals in the sequence) and the SIRT ( 4 produced intervals, 4 durations $\times 1$ interval in the sequence). We used the algorithm (Hanes et al., 1995) to detect activations above randomness, as follows. The mean discharge rate $(r)$ was computed for the entire recording session of the cell (i.e., the four task combinations). The first two consecutive spikes that had a mean discharge rate greater or equal to $r$ were found, and the time between these two spikes was defined as the initial $T$ value. Then, the next spike was identified, and the interspike interval (ISI) between this and the previous spike was added to $T$. The corresponding SI was calculated. This was repeated until the end of the spike train, and the spike at the end of the interval $T$ with the maximum SI was defined as the end of the burst. Next, the SI was calculated for the interval $T$ from the last to the first spike. Then, the spikes from the beginning were removed until the end of the spike train, computing the corresponding SI in each step. The spike at which SI was maximized was defined as the beginning of the burst. All produced intervals that showed a burst larger than $80 \mathrm{~ms}$ and a SI $p<0.05$ were considered as having a significant activation. If this criterion was not fulfilled, it was assumed that there was no response for that target duration/ordinal sequence combination. We found cases with more than one significant burst inside a produced interval, and in this situation we computed the average discharge rate for the two periods of activation.

Interval and sequence selectivity. We recorded 993 neurons during the performance of the four task combinations (see above). The activity of these cells was subjected to two initial analyses. The first one was per- 
formed to determine the cells with significant differences in their response magnitude across durations, for each produced interval in the rhythmic sequence of the SCT, and for the single reproduced interval of the SIRT. The corresponding ANCOVA used the discharge rate computed from the Poisson train analysis as the dependent variable, the discharge rate during the key holding control epoch as the covariate, and the target interval as the factor. The second analysis was performed to determine the cells with significant changes in activity across duration, ordinal sequence, or both parameters during the SCT. This analysis consisted of a two-way ANOVA where the discharge rate computed from the Poisson train analysis was the dependent variable and the target interval $(450,550,650,850$, and $1000 \mathrm{~ms})$ and the ordinal sequence (one to six produced intervals) were the factors.

Classification of ordinal sequence or task phase selectivity. Cells with significant ANOVA effects on Sequence or Interval $\times$ Sequence interaction were segregated in two functionally distinct cell populations, namely ordinal- or phase-selective neurons, using K-means clustering. This procedure partitioned neural responses into 13 clusters, where each cell was assigned to the cluster with the nearest mean (Johnson and Wichern, 1998). The initial means of the clusters were the following:

$$
\begin{aligned}
& \mu_{1}=(1,0,0,0,0,0) \\
& \mu_{2}=(0,1,0,0,0,0) \\
& \mu_{3}=(0,0,1,0,0,0) \\
& \mu_{4}=(0,0,0,1,0,0) \\
& \mu_{5}=(0,0,0,0,1,0) \\
& \mu_{6}=(0,0,0,0,0,1) \\
& \mu_{7}=(1,1,0,0,0,0) \\
& \mu_{8}=(0,1,1,0,0,0) \\
& \mu_{9}=(0,0,1,1,0,0) \\
& \mu_{10}=(0,0,0,1,1,0) \\
& \mu_{11}=(0,0,0,0,1,1) \\
& \mu_{12}=(1,1,1,0,0,0) \\
& \mu_{13}=(0,0,0,1,1,1)
\end{aligned}
$$

where $\mu_{1}-\mu_{6}$ correspond to the clusters that responded only to one element of the six interval sequence; $\mu_{7}-\mu_{11}$ to the clusters that responded to two consecutive elements of the sequence; and $\mu_{12}$ and $\mu_{13}$ to the synchronization and continuation phase, respectively. The normalized discharge rate of the Poisson train analysis for each ordinal element, across durations and trials (a total of 30 trials for each ordinal element), was used as the dependent variable. A one-way multivariate ANOVA was performed using the normalized responses of cells across each ordinal element as dependent variables and the clustering results as factors. The results showed that the 13 clusters were significantly different from each other $\left(\chi_{(72)}^{2}=3.61 \times 10^{3} ; p<0.00001\right)$.

Gaussian regression. A Gaussian function was fitted to the cell activity in MPC to determine the tuning to duration. These fittings were performed only on the cells with a significant effect on the corresponding ANOVA (see above). The discharge rate computed from the Poisson train analysis for each produced interval during the SCT or the SIRT was treated as the dependent variable in a nonlinear regression where the target duration was used as independent variables in the following equation:

$$
f(s)=h e^{-\left(\frac{s-s_{p}}{k}\right)^{2}},
$$

where $f(s)$ corresponds to the discharge rate associated with a particular value of the independent variable $s, h$ is the parameter of maximum height, and $k$ is the parameter of dispersion. $s_{p}$ corresponds to the pre- ferred interval. The regression was performed for each of the six elements of the SCT sequence and for a single produce interval in the SIRT. The function was fitted using the least squares method following a genetic algorithm implemented in Matlab (version 7.3.0.267; Mathworks). A detailed analysis of the residuals was performed (Draper and Smith, 1981 ), and the $R^{2}$ was calculated. Furthermore, the significant level of the $R^{2}$ was assessed using a bootstrap technique as follows. First, the firing rate of the 25 total trials collected (five repetitions, five durations) was permuted to get five random mean firing rates. Second, a curve was fitted to these data, and the $R^{2}$ was computed. This procedure was repeated 1000 times, and the distribution of $R^{2}$ values was saved. Finally, if the $R^{2}$ of the original regression was larger than the value at 0.95 of the bootstrap $R^{2}$ distribution, the regression was considered significant (Merchant et al., 2008c). Nonsignificant ( $p \geq 0.05$ ) or out-of-bounds fits were excluded from the results.

Tuning dispersion measure. We used the half-width dispersion, $k_{50}$, at the midpoint of the tuning magnitude as the consistent measure of tuning dispersion. The corresponding equation was as follows:

$$
k_{50}=\sqrt{\ln 2 k}
$$

Double-Gaussian regression. A double-Gaussian was fitted to the cell activity in MPC to determine produced duration and sequence-order tuning during the SCT using the following equation:

$$
f(I, S)=h e^{-\left(\frac{I-I_{p}}{k_{I}}\right)^{2}-\left(\frac{S-S_{p}}{k_{S}}\right)^{2},}
$$

where $h$ is the parameter of maximum height and $k_{I}$ and $k_{S}$ are the parameters of dispersion for interval and sequence, respectively. $I_{p}$ and $S_{p}$ correspond to the preferred interval and preferred sequence order, respectively. The significant level of the $R^{2}$ was assessed using the above bootstrap technique. Again, nonsignificant ( $p \geq 0.05$ ) or out-of-bounds fits were excluded from the results.

Bayesian decoding. We used a Bayes analysis approach to address the following problem: given the firing rates of cell populations tuned to both the interval and the ordinal structure of the SCT, how can we optimally infer the sequential and temporal behavior of the animals in the task? The basic method assumes that we know the encoding functions $f_{1}(I, S)$, $f_{2}(I, S), \ldots f_{\mathrm{N}}(I, S)$ are associated with the produced duration/sequence order for a population of $n$ cells from Equation 5. Given the discharged rate ( $r$, based on the Poisson train analysis), fired by the cells within a specific produced duration-sequence combination, the objective was to compute the decoded probability distribution for both task parameters, across trials with similar temporal behavior throughout the six produced intervals.

Let the vector $x=(I, S)$ be the duration-sequence combination and the vector $r=\left(r_{1}, r_{2}, \ldots, r_{\mathrm{N}}\right)$ be the discharge rate of our recorded cells within this period. The reconstruction is based on the following standard Bayes equation for conditional probability:

$$
p(x \mid r)=\frac{p(r \mid x) p(x)}{p(r)} .
$$

The goal is to compute $p(x \mid r)$, that is the probability for the duration-sequence parameters to be at the value $x$, given the firing rates $r . p(x)$ is the durationsequence probability, which was dependent on the overall distribution of the produced durations by the monkey and the constant sequential structure of the SCT. The probability $p(r)$ for the occurrence of the firing rate $r$ is equal to the sum of the conditional probability $p(x \mid r)$ over all $p(x)$. Thus, the key step is to evaluate $p(x \mid r)$, which is the probability that the firing rate $r$ occurred given that we know the duration-sequence combination $x$. It is intuitively clear that this probability is determined by the estimated firing rates from Equation 5. More precisely, if we assume that the cell activity has a Poisson distribution and that the different cells are statistically independent of one another, then we can obtain the following explicit expression:

$$
p(r \mid x)=\prod_{i=1}^{N} \frac{f_{i}(x) T}{\left(r_{i} T\right) !} e^{-f_{i}(x) T}
$$


where $f_{\mathrm{i}}(x)$ is the average predicted firing rate of cell $i$ of a population of $n$ cells, $x$ is the duration-sequence parameter, and $T$ is an arbitrary time window (500 $\mathrm{ms}$ in this case).

The Bayesian reconstruction method uses Equation 7 to compute the probability distribution $p(x \mid r)$ for the parameter's combination $x$ given the firing rate $r$ for all cells associated with that $x$. Then, once Equation 6 is solved, we consider the maximum value of the computed probability distribution as the decoded duration-sequence parameter (Merchant and Perez, 2009). In other words:

$$
\hat{X}_{M A P}=\arg \max _{x} p(x \mid r)
$$

To systematically decode both the duration of the produced intervals and the sequential order of the SCT, we used groups of trials to compute $p(x \mid r)$ where the temporal behavior on the monkeys was similar and where the activity of cells during this behavior was significantly tuned according to Equation 5. Hence, the SCT trials during cell recording were divided into four classes using the following procedure. First, the medians of the produced durations for each of the three intervals in the synchronization and the three intervals in the continuation phase were computed for the entire database. Then, each of the six consecutive intervals of a trial were called "short" or "long" if their value was below or above the median of the corresponding six distributions. Finally, the synchronization phase of a trial was classified as "preferential short" if two or three of its produced intervals were short, or as "preferential long" if two or three of its produced intervals were long. The same criteria were used for the continuation phase. Consequently, the four classes of trials were (1) synchronization short-continuation short, (2) synchronization short-continuation long, (3) synchronization long-continuation short, and (4) synchronization long-continuation long.

For each of the four classes, we performed 1000 decodifications using permuted populations of 200 trials to avoid a population-size effect in the reconstructed values. Since this decoding method used groups of trials with similar timing performance across neurons that were not necessarily recorded simultaneously, we also performed decoding with crossvalidation to access possible overfittings. In this case, 1000 decodifications for each condition were performed using permuted populations of 150 cells (from the total number of neurons, namely 246 for visual and 216 for the auditory conditions) and a leaving-one-out cross-validation algorithm, with the purpose of sampling the reconstruction accuracy (variance and bias; Dayan and Abbott, 2001, their Eqs. 3.38 and 3.39) within the overall cell population. The results of the cross-validation method were very similar to the decoded values shown in Figure 5, indicating high correlation values between the two decoding measures (Auditory Duration: $r=0.82, p<0.0001$; Auditory Ordinal-Sequence: $r=0.99, p<0.0001$; Visual Duration: $r=0.84, p<0.0001$; Visual Ordinal-Sequence: $r=0.98, p<0.0001$ ). Therefore, the results showed in Figure 5 are not the result of data overfitting.

The bias between the decoded and the behavioral parameters, shown in Figure 6C-F, was evaluated using the standard methods (Johnson and Wichern, 1998). On the other hand, Figure 6 shows the mean and the two-dimensional variability of all decoded values. The two-dimensional variability was characterized using the bivariate normal distribution in the form of an ellipse. This ellipse is centered at the $x-y$ (sequenceduration) mean, and the length of its axes is proportional to the square root of the two eigenvalues of the $x-y$ variance-covariance matrix. The two axes are orthogonal and are equivalent to the variances along each axis (i.e., the larger axis corresponds to the axis of larger variance). We scaled the axis using the constant where there is the upper $(100 \alpha)$ th percentile of the $\chi^{2}$ distribution with $k$ degrees of freedom. This leads to an ellipse that contains the $(1-\alpha) \times 100 \%$ of the distribution probability, where $\alpha=0.69$ that corresponds to the SD of the distribution (Fig. 5). Finally, the orientation of the ellipse was defined by the angle $\theta$ that was equal to the arctangent of the $x$ and $y$ elements of the eigenvector from the larger eigenvalue (Johnson and Wichern, 1998).

Cell stability during the performance of the four tasks. We used previously validated criteria to assess the single-unit stability during the performance of the tasks by measuring the similarity of the average spike waveforms and the ISI histograms (ISIHs) (Dickey et al., 2009). For the case of the ISIH, we used a score that compares the overall shape of the ISIH for two tasks. The ISIH shape for each task was modeled using a mixture of three log-normal distributions, where each distribution can be considered as comprising a fast (centered on $2.5 \mathrm{~ms}$ ), a medium (30 $\mathrm{ms}$ ), and a slow (1 s) ISIH component. The mixture model was fitted using an expectation-maximization algorithm (Hastie et al., 2001). Hence, each ISIH was described by eight parameters: the mean and SDs of the three components and the mixing probabilities of the first two components (the last is not needed because the probabilities sum to 1). Then, the similarity score $I$ between the ISIHs of two tasks was defined as follows:

$$
I(A, B)=\sqrt{\sum_{i=1}^{8} \frac{\left(A_{i}-B_{i}\right)^{2}}{\sigma_{i}^{2}}},
$$

where $A$ and $B$ are the two sets of eight parameters and $\sigma_{\mathrm{i}}$ are the normalizing factors obtained from the variance of the eight parameters for a set of sample data. The data come from the ISIHs of the four tasks of the cell in Figure $7 A-D$. Therefore, a similarity score $I$ close to zero indicates stability, whereas a high value indicates instability of the cell between the two tasks. The stability threshold used here was 10.5 , which was reported previously as an appropriate value in chronic single-cell recordings (Dickey et al., 2009).

A total of 762 cells showed a similarity score $I$ below the 10.5 threshold (Dickey et al., 2009) in at least two consecutive tasks and were considered stable between the task pairs with $I$ scores below that threshold. Thus, the difference in preferred intervals across tasks was computed for a population of 668 neurons that showed both a significant Gaussian fitting for interval tuning and stable responses according to the similarity score $I$ for pairs of consecutive tasks combinations.

Bootstrap for preferred intervals across tasks. We performed a bootstrap analysis to test whether the percentage of cells with similar preferred intervals (PI difference $<150 \mathrm{~ms}$ ) was above chance for different task pairs. We built 5000 bootstrap populations, by selecting randomly the PI of the cells within each task pair [i.e., auditory SCT (SCTa) vs visual SCT $(\mathrm{SCTV})]$ and computed the PI difference for each randomly selected pairs. Then, we measured the percentage of cells with similar PIs (PI difference $<150 \mathrm{~ms}$ ) in tuning in these bootstrap populations and compared it with the original percentage of cells with similar PIs. We found that for all the task pairs [SCTa-SCTv, auditory SIRT (SIRTa)visual SIRT (SIRTv), SCTa-SIRTa, and SCTv-SIRTv] the probability that the bootstrapped populations showed a similarity in PI between tasks that was equal or above the original PI similarity was always below $p=0.05$.

\section{Results}

\section{Behavioral performance}

We investigated how MPC neurons encoded the temporal structure of different tapping behaviors that varied in the number of produced intervals and the modality used to drive temporal processing. We trained two monkeys in a SCT and a SIRT (Zarco et al., 2009). In the SCT, the monkeys were required to push a button each time stimuli with a constant interstimulus interval were presented, which resulted in a stimulus-movement cycle (Fig. 1A). After four consecutive synchronized movements, the stimuli stopped, and the monkeys continued tapping with the same interval for three additional intervals (Fig. 1A). SIRT started with training trials where the monkey tapped twice on a button to reproduce an instructed target interval, followed by testing trials where a go signal triggered the reproduction of the same interval (Fig. 1B). Brief auditory or visual interval markers were used during both tasks, and the range of target intervals was from 450 to $1000 \mathrm{~ms}$. The monkeys were able to accurately produce the target intervals, showing an average underestimation of $\sim 50 \mathrm{~ms}$ across durations and modalities, during the testing phase of SIRT and both task phases of the SCT (Fig. 1C,D). In addition, we analyzed the temporal variability of the monkeys' tapping perfor- 
mance, which was defined as the SD of the individual interresponse intervals (Merchant et al., 2008a) (see Materials and Methods). Temporal variability increased linearly as a function of duration in SIRT and the synchronization and continuation phases of SCT (Fig. 1E,F). The timing behavior was similar for the auditory and visual interval marker conditions (Fig. $1 C-F ; t$ test for constant error and temporal variability, $p>0.05)$. These findings show that the monkeys had a remarkably accurate timing performance in complex temporal tapping tasks. Furthermore, the data show a temporal variability that followed the scalar property of interval timing, a property that has been documented in many species and temporal tasks (Gibbon et al., 1997).

\section{Generalities on the neurophysiology of interval production}

A large number of parameters were used in the present experimental design to test the existence of a single or multiple neural clocks in a cortical area that has been associated with temporal processing in functional imaging and neurophysiological studies (Mita et al., 2009; Wiener et al., 2010; Merchant et al., 2011). Our results indicated for the first time that cells in MPC were tuned to intervals in the hundreds of milliseconds range, showing an orderly variation in discharge rate as a function of the produced duration in the SIRT and SCT. Thus, the present study focuses on the comparison of the interval tuning properties of cells between tasks [which involve the time production of one (SIRT) or six intervals (SCT)] and the modality used to define the intervals (auditory vs visual). In the initial part, we describe the interval tuning properties of neurons during SCT and SIRT. Then, we describe strong interaction between the neural signals associated with the representation of the produced interval and the organization of sequential motor behavior during the SCT. Next, we determine the differences in preferred intervals across tasks and modalities, showing interval-tuning invariance in a large population of cells. Finally, we provide evidence for a possible gain mechanism for encoding the total number of intervals in a sequence of rhythmic movements.

\section{Interval tuning in SCT and SIRT}

We recorded from 993 randomly selected MPC cells that showed stable responses during the performance of the four task combinations (Fig. 1G). An initial analysis was performed to determine the cells with significant differences in their response magnitude across interval durations, for each produced interval in the rhythmic sequence of the SCT, and for the single reproduced interval of the SIRT. The corresponding ANCOVA used the discharge rate as the dependent variable, the discharge rate during the key holding control epoch as the covariate, and the target interval as the factor. It is important to emphasize that the duration of target intervals varied systematically in our tasks, and therefore we could not use a fixed temporal window to compute the discharge rate of the cells. As an alternative, we used the Poisson train analysis (see Materials and Methods) to identify the periods of cell activation within each produced interval. Table 1 shows the number of cells with a significant effect on interval duration in the ANCOVA for the four task combinations. It is evident that a large percentage of cells showed modulations in activity for different durations in the SCT and SIRT, with a small bias toward visual marker conditions in both tasks.

We fitted Gaussian functions to the discharge rate of cells as a function of interval on the cells with significant ANCOVA effects for Interval. Most of these cells showed significant Gaussian tuning (Table 1), and therefore they were considered interval-

\section{Table 1. Number of neurons with a significant effect on target interval in the ANCOVA and a significant Gaussian fit for interval across the four task combinations}

\begin{tabular}{lll}
\hline Task & ANCOVA & Gaussian fit \\
\hline SCT auditory & $521(52.5)$ & $440(84.5)$ \\
SCT visual & $570(57.4)$ & $487(85.4)$ \\
SIRT auditory & $304(30.6)$ & $229(75.3)$ \\
SIRT visual & $317(31.9)$ & $232(73.2)$ \\
\hline
\end{tabular}

Numbers in parentheses correspond in the ANCOVA to the percentages from the 993 studied neurons and in the Gaussian fits to the percentages from the neurons with significant effects in the ANCOVA.

selective cells (Fig. 2). Figure 2, $A$ and $B$, shows the raster of a cell tuned for a long duration during both the SCT and SIRT in the auditory interval maker condition. The preferred interval of the cell was around $880 \mathrm{~ms}$ in both tasks, as shown in Figure $2 C$ for the respective Gaussian fittings.

Although a wide range of preferred intervals was represented in the population of interval-selective cells, the distribution of preferred intervals showed a bias toward long intervals in the four task combinations (Fig. 2D-G). In addition, the half-height tuning dispersion was skewed toward low dispersions (see Fig. $2 \mathrm{H}$ for the SCT in the visual condition; the tuning dispersion for the other three task combinations is not depicted but showed a similar trend). The results suggest that MPC cells showed an orderly variation in discharge rate as a function of the produced duration in the SIRT and SCT for auditory and visual interval marker conditions, where all possible preferred intervals are represented in the cell population, even though there was a clear bias toward long durations.

\section{Cell encoding for interval and ordinal sequence during the SCT}

A large group of neurons during the SCT showed activity that varied significantly (two-way ANOVA) with the interval, the task sequence ( six elements, three in the synchronization and three in the continuation phase), or both parameters during this task (Auditory = Interval, 475 of 993 cells; Sequence, 461; Interval $\times$ Sequence, 172; Visual = Interval, 494; Sequence: 511 ; Interval $\times$ Sequence, 237). These findings suggest that MPC shows strong neural signals for both the sequential and the temporal structure of the SCT. Therefore, the next step was to characterize the encoding properties of MPC neurons for these task parameters, focusing first on sequence, then on the interaction between ordinal sequence and duration.

Cells with significant ANOVA effects on Sequence or Interval $\times$ Sequence interaction were segregated in two functionally distinct cell populations according to their responses to the sequential order of the SCT using a clustering algorithm (see Materials and Methods). The first group may encode the ordinal structure of the task sequence, since these cells responded during one or two consecutive elements of the six interval sequence (Table 2; Fig. 3A-C). These cells were considered ordinal-selective cells, and they indicate that the well known ordinal sequential movement activity in MPC (Tanji, 2001) is also present during the execution of the SCT. In contrast, the second group consisted of cells that were active during the synchronization or continuation phase of the SCT (Table 2; Fig. $3 A, D$ ). This type of phaseselective response may be associated with the temporal information processing during sensory-guided (synchronization) or internally driven (continuation) cyclic movement production.

The interaction between the neural signals associated with the organization of sequential motor behavior and the representa- 

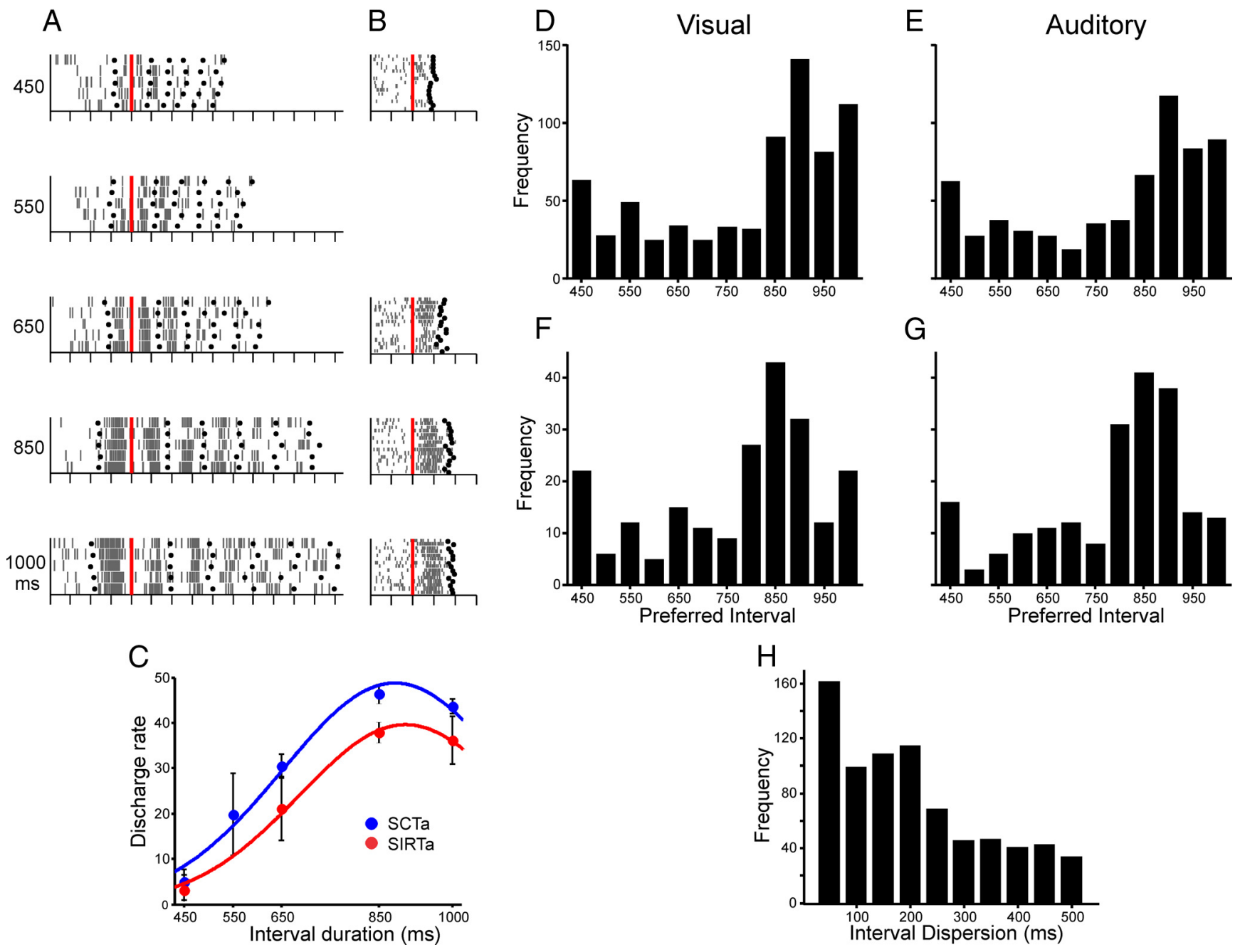

Figure 2. Cell tuning for interval during the SCT and SIRT. $A$, Responses of an interval-tuned cell with a long preferred interval during the first two elements of the synchronization phase of the SCT in the auditory marker condition. The raster histogram is aligned (red line) to the second tap of the synchronization phase. Black dots correspond to tapping times. $\boldsymbol{B}$, Responses of the interval-tuned cell in $\boldsymbol{A}$ with a long preferred interval during the SIRT in the auditory condition. The raster histogram is aligned (red line) to the first tap. $\boldsymbol{C}$, Tuning functions for the cell in $\boldsymbol{A}$ and $\boldsymbol{B}$, where the mean \pm SEM of the discharge rate is plotted as a function of the target interval. The continuous lines correspond to the significant Gaussian fits. SCTa, SCT auditory (blue line); SIRTa, SIRT auditory (red line). $\boldsymbol{D}$, Histogram of preferred intervals in the visual condition for cells with significant interval tuning during the SCT (median, $842.3 \mathrm{~ms}$ ). $\boldsymbol{E}$, Histogram of preferred intervals for tuned cells during the SCT in the auditory condition (median, $835.7 \mathrm{~ms}$ ). $\boldsymbol{F}$, Histogram of preferred intervals for tuned cells during the SIRT in the visual condition (median, $821.3 \mathrm{~ms}$ ). $\mathbf{G}$, Histogram of preferred intervals for tuned cells during the SIRT in the auditory condition (median $808.5 \mathrm{~ms}$ ). $\boldsymbol{H}$, Histogram of half-height tuning dispersion of the cells in $\boldsymbol{D}$ (median, $179.4 \mathrm{~ms}$ ).

Table 2. Number of neurons whose responses were classified as sequence selective to one or two consecutive elements of the six-interval sequence or phase selective (synchronization or continuation) or were unclassified during the visual and auditory conditions of the SCT

\begin{tabular}{llc}
\hline & SCT visual & SCT auditory \\
\hline Sequence 1 & 241 & 199 \\
Sequence 2 & 185 & 193 \\
Phase & 81 & 51 \\
Unclassified & 42 & 52 \\
\hline
\end{tabular}

tion of the actual produced interval during the SCT (Fig. 4) was described using double-Gaussian regressions on the ordinalselective cells. In this case, however, we computed the regressions using the duration of the produced intervals during the task execution, instead of the target intervals with which the animals were instructed, to capture the relationship between neural activity and temporal performance (nevertheless, similar results were obtained with target interval as the independent variable). We found that $221(56.4 \%, 221$ of 392$)$ of the ordinal-selective cells in the auditory condition and 250 (58.7\%, 250 of 426) in the visual marker condition were significantly tuned to both variables during the SCT. Figure 4, $A$ and $C$, shows the raster plots and doubletuning curve, respectively, for a cell with a short preferred interval and a preferred sequence order around the first continuation interval, whereas Figure 4, $B$ and $4 D$, corresponds to a cell that was sharply double-tuned with a long preferred interval and a preferred sequence order around the second continuation interval. The density of preferred interval and preferred sequence order for all the significant double-tuned cells showed a bias toward longer durations, as well as a bias for the synchronization phase in the auditory condition (Fig. $4 F$ ) and a bias for the continuation phase in the visual condition (Fig. 4E). In addition, some cells in these distributions showed a preferred sequence order during the transition between phases (synchronization-continuation), which could imply that these cells could be phase transition detectors instead of sequence-selective cells. Nevertheless, all possible combinations of duration/sequence order were encoded in the population in both interval marker conditions. Figure 5 shows the $\chi^{2}$ values between the predicted and the actual re- 


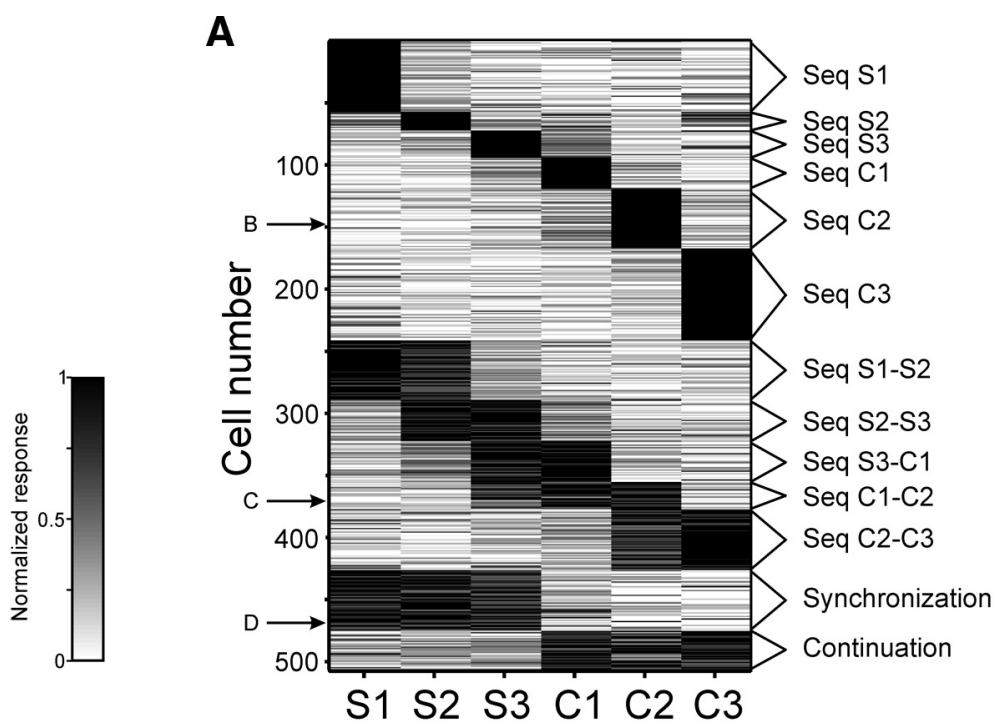

B

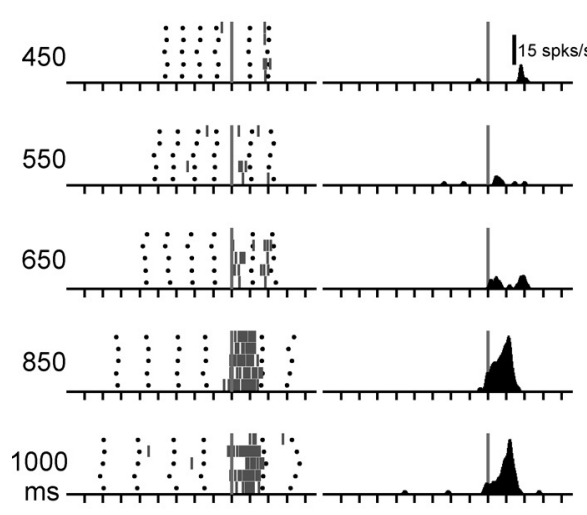

C

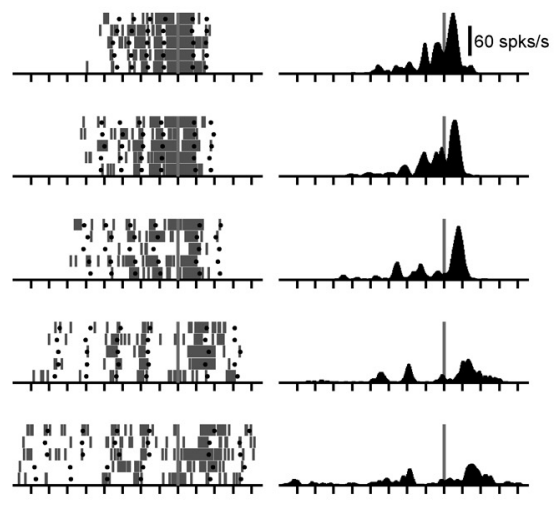

D

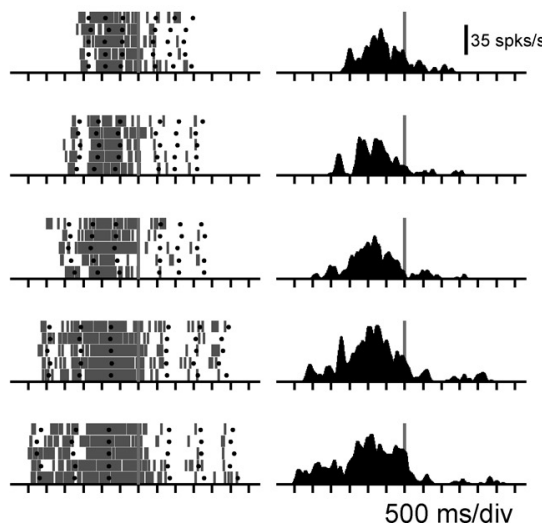

Figure 3. Cell selectivity to the sequential order and task phase during the SCT. A, Normalized activity for a population of neurons with significant effects on sequence (Seq; ANOVA) that were classified as sequence selective to one, two, or three consecutive elements of the SCT using K-means clustering. Each row corresponds to one cell, and each column corresponds to one element of the SCT sequence. The letters with the arrows correspond to the raster of the cells below. $\boldsymbol{B}$, Responses of a cell with a preferred sequence order to the second continuation interval. This cell is also tuned

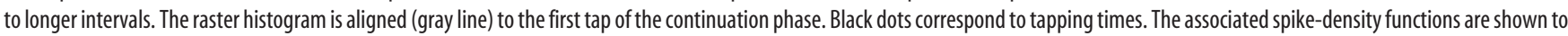
the right. C, Cell that shows a selective response for the first and second intervals of the continuation phase, which also shows a preference for short intervals. The raster histogram is aligned to the first tap of the continuation phase. $\boldsymbol{D}$, Responses of a cell with selective activity during the synchronization phase of the SCT. The raster histogram is aligned to the last tap of the synchronization phase.

sponses of individual cells for the double-Gaussian against the single-Gaussian model for interval duration using the six elements of the SCT sequence. It is evident that most cells showed better fittings for the double-Gaussian function, in the visual (Fig. 5A) and auditory (Fig. 5B) conditions. Overall, these analyses suggest that the MPC shows a simultaneous representation of duration and sequential order during the execution of a rhythmic task such as the SCT.

It is important to mention that some cells that were phase selective on the cluster analysis were also significantly tuned to interval (Auditory: 23.5\%, 12 of 51; Visual: 37\%, 30 of 81; see Fig. $7 A, B)$, suggesting that MPC can multiplex interval information with the number of elements in a sequence or with the context in which the tapping rhythms are generated (i.e., sensory driven or internally generated).

\section{Decoding the temporal-sequential structure of the SCT}

Once we determined the dependence of the neural responses on the ordinal sequence of the SCT and the durations produced by the monkeys during task execution, we used a Bayes analysis approach to address the inverse problem: given the firing rates of double-tuned cells, how can we optimally infer the sequential and temporal behavior of the animals in the task? Figure 6, $A$ and $B$, shows the mean (black dots) and two-dimensional variance (ellipses) of the decoded values by populations of double-tuned cells recorded during trials with similar produced lengths (see Materials and Methods), for each target interval and across the sixinterval sequence of the task, for the auditory and visual conditions. In addition, the colored squares in Figure 6, $A$ and $B$, depict the mean \pm SD of the produced intervals in the trials used for the decoding. The decoded values were accurate, particularly for the synchronization phase in the auditory condition and the continuation phase in the visual condition, across the five target durations ( $t$ tests between phases, $p<0.05$ ). Furthermore, the decoding was more accurate for 650 and $850 \mathrm{~ms}$. These findings, then, are in accordance with the bias in the distribution of preferred intervals and ordinal sequence shown in Figure 4, $E$ and $F$. Thus, these results suggest that MPC shows a temporal process- 
A
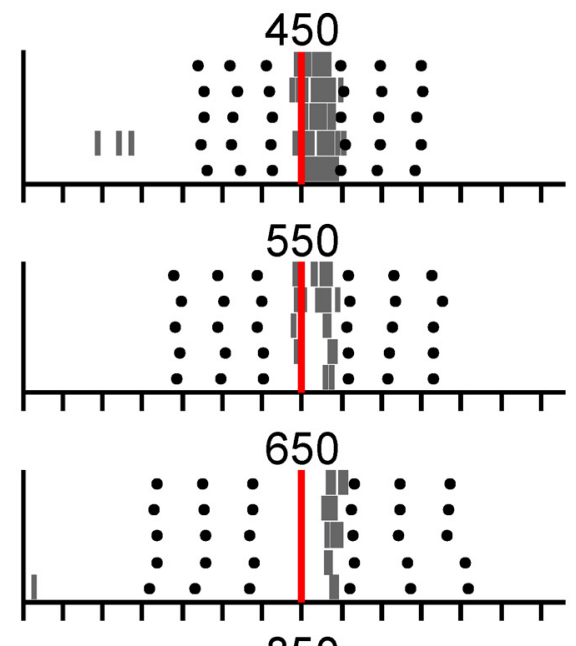
850

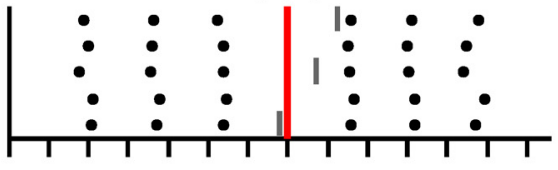
1000

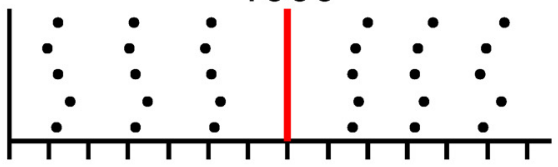

C

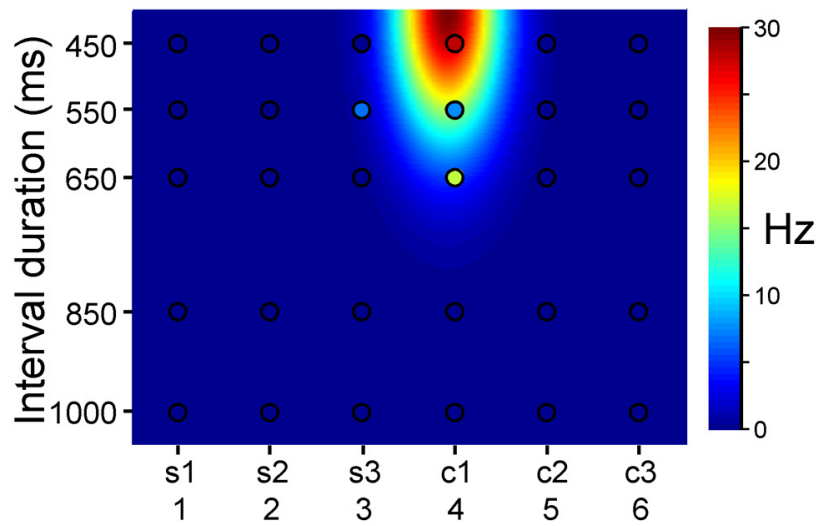

E

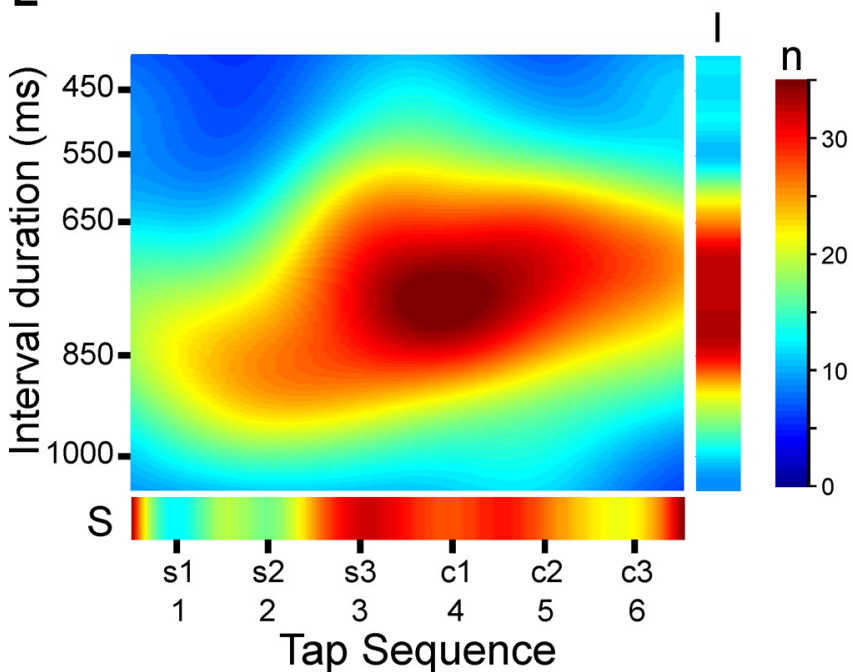

B

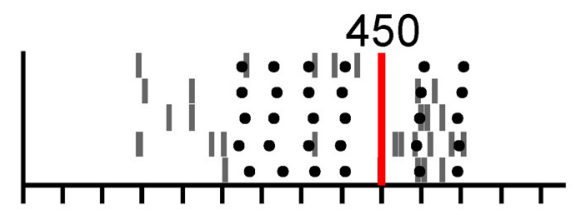

550

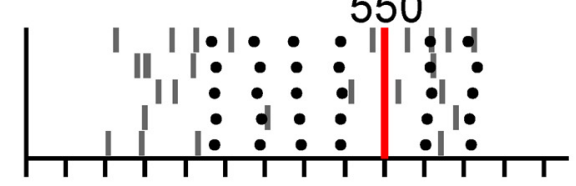

650

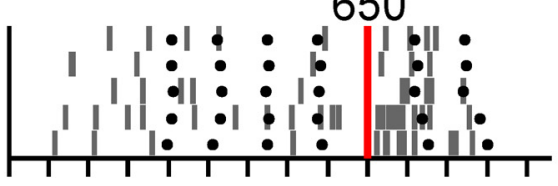

850

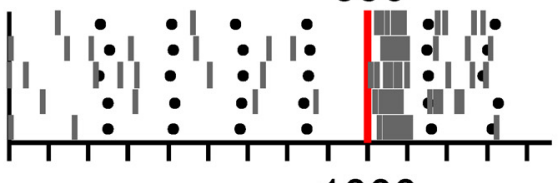

1000

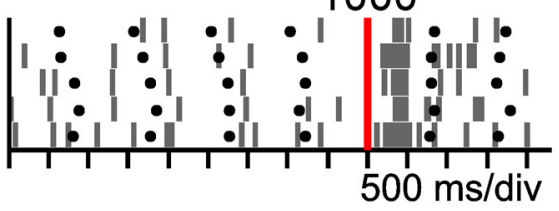

D

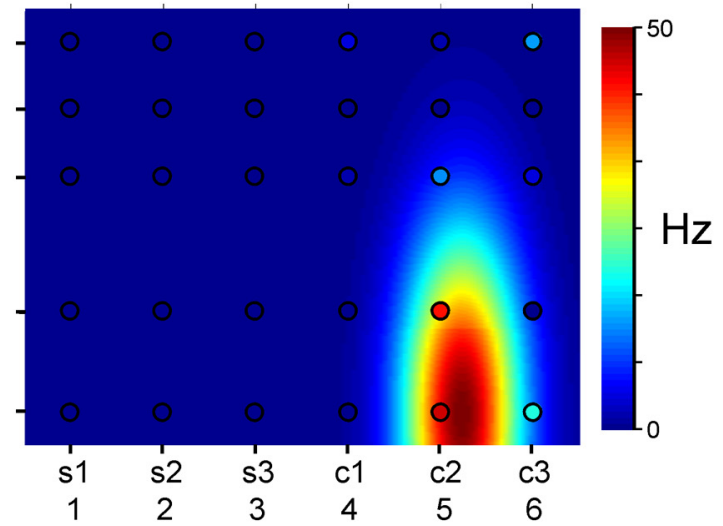

F

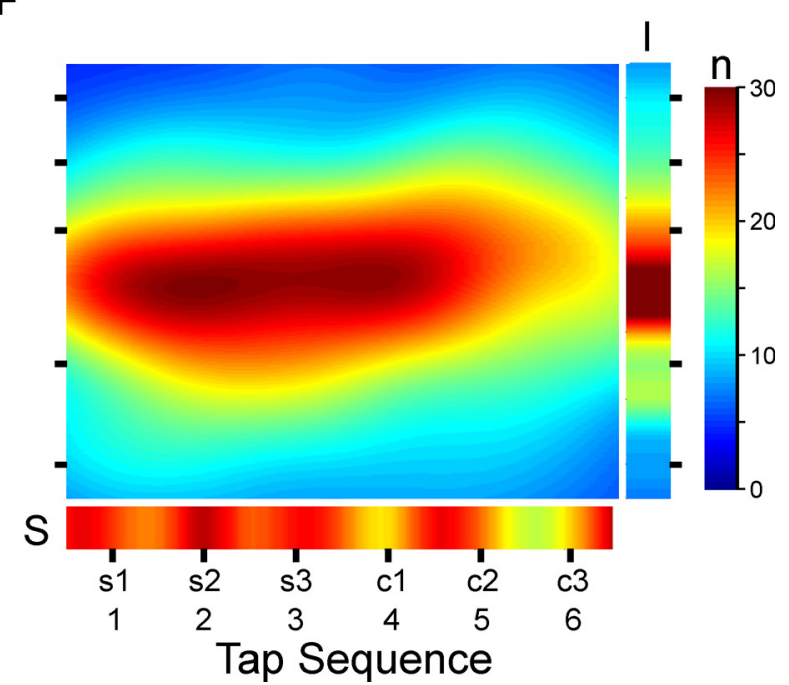


A

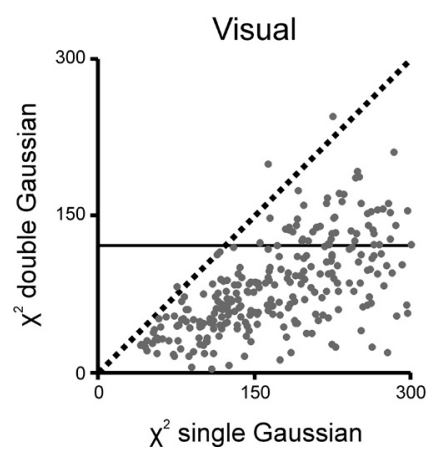

B

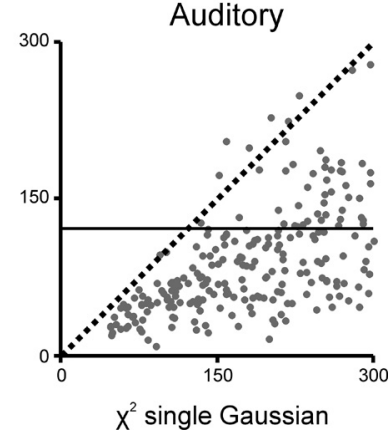

Figure 5. Comparison of $\chi^{2}$ between single- and double-Gaussian fittings for cells with significant effects of duration in the ANCOVA for the visual $(\boldsymbol{A})$ and auditory $(\boldsymbol{B})$ conditions. $X^{2}=\sum_{i} \frac{\left(O_{i}-E_{i}\right)^{2}}{E_{i}}$, where $O_{\mathrm{i}}$ is observed discharge rate and $E_{\mathrm{i}}$ is expected discharge rate for trial $i$. For single-Gaussian fittings, the discharge rate across trials and sequence order was the dependent variable, and target interval was the independent variable, using Equation 3. For the double-Gaussian fittings, the discharge rate computed for each interval and sequence order across trials was the dependent variable, and interval and sequence order were the independent variables, using Equation 5 . In both fittings, we used 150 trials: 5 intervals $\times 6$ sequence orders $\times 5$ trials. The dotted line corresponds to the diagonal, and below the continuous horizontal, the cells showed a significant fitting on the $\chi^{2}$ test $(p<0.05)$. It is evident that the double-Gaussian model showed smaller $\chi^{2}$ and better fittings than the singleGaussian model.

ing that depends on the modality of the interval markers used during the synchronization phase. Furthermore, the bias between the decoded and produced durations showed a pattern of overestimation for short durations and of underestimation for long intervals for both marker modalities, following the same trend of the monkey behavior (compare Figs. $1 C, D, 6 C, D$ ). Figure $6 F$ shows for the auditory marker condition a small underestimation for the decoded sequential-order values during the continuation, whereas Figure $6 E$ shows a small overestimation between the decoded and sequential order values during the synchronization. Overall, the results suggest that cell populations in MPC have information to represent the sequential-order as well as the duration of intervals during rhythmic behavior. This neural population signal may correspond to a two-dimensional representation of the ordinal and temporal structure of movement sequences that could be present during music execution, for example.

\section{Interval tuning in MPC across timing contexts}

Next, we wanted to test whether a common timing mechanism or a set of context-dependent neural clocks could explain the behav-

\section{$\leftarrow$}

Figure 4. Cell tuning to interval and sequential order during the SCT. $\boldsymbol{A}$, Responses of a double-tuned cell with a short preferred interval and a sharp preferred sequence order around the first continuation interval. The raster histogram is aligned (red line) to the first tap of the continuation phase. $\boldsymbol{B}$, Responses of a sharply double-tuned cell with a long preferred interval and a preferred sequence order around the second continuation interval. The raster is aligned (red line) to the second tap of the continuation phase. $C, D$, Double-Gaussian tuning functions for the cell responses depicted in $\boldsymbol{A}$ and $\boldsymbol{B}$, respectively. The dots correspond to the mean discharge rate for each duration-sequence order combination. The color code represents the discharge rate (hertz) of the cells. $\boldsymbol{E}, \boldsymbol{F}$, Distribution of the dual preferred interval/sequence order for the cell population with significant Gaussian fits in the visual and auditory marker conditions, respectively. S, Distribution of preferred ordinal sequence; I, distribution of preferred interval. The color code represents the number ( $\mathrm{n}$ ) of double-tuned cells in different duration and sequence order combinations.

ior of interval-selective cells, comparing their tuning properties not only between the visual and auditory marker conditions, but also among the SCT and SIRT for the same set of intervals. Figure $7 A-D$ shows the activity profiles of a cell that was tuned with preferred intervals around $850 \mathrm{~ms}$ during the SCT and SIRT for both interval marker modalities. The similarity in preferred intervals among tasks in this cell (Fig. 7E) supports the notion that interval tuning is a representation of time that may be used in a variety of behaviors and different sensory modalities. It is important to mention that the shapes of both the spike waveform (Fig. $7 A-D$, bottom insets) and the ISIHs of this cell were very similar between tasks, indicating that the recordings during the four tasks were stable and that the tuning properties across tasks belonged to the same cell (data not shown). Then, we compared the interval tuning properties of 668 cells across tasks. These cells were considered stable between tasks using various criteria, which included the average spike waveforms and the shape of the ISIHs, and were tuned to the interval in at least one pair of the four task combinations (see Materials and Methods) (Dickey et al., 2009). Interestingly, $61.8 \%$ (412 of 668) of the cells showed similar preferred intervals (with a difference below $150 \mathrm{~ms}$ ) across the different combinations of tasks pairs (Fig. 7F; Table 3). Finally, Figure $7 \mathrm{G}$ shows the density of preferred intervals for cells that were interval selective for different combinations of task pairs. This graph shows that, although there was the bias toward long durations, there was a group of cells that showed a similar preferred interval across tasks, particularly for the shorter and longer preferred intervals.

We performed a more detailed analysis of the cells' tuning properties, using the cumulative distributions of the difference in preferred intervals for the same cells between specific tasks pairs, to determine whether the number of produced intervals (one for SIRT and six for SCT) and the modality of the interval marker produced systematic drifts in the cells' preferred intervals.

The results corroborate that more than half of the MPC cell population showed small differences in their preferred interval across behavioral contexts (Fig. 8A; Table 4). In addition, the cumulative distributions of the preferred interval differences between same tasks but different modalities (Fig. 8A, SCTa - SCTv in blue and SIRTa - SIRTv in red) were shifted to the left with respect to the distributions of different tasks but same modality (Fig. 8A, SCTa - SIRTa in green and SCTv - SIRTv in orange; Table 5). Finally, a bootstrap analysis showed that the similarity in preferred intervals (with a difference below $150 \mathrm{~ms}$ ) across these tasks pairs was above chance $(p<0.05$; see Materials and Methods).

To assess the similarities in cell responses between tasks pairs across all the tested durations and not only the preferred interval, we performed a linear regression using the discharge rates of the same cells across durations between different pairs of tasks. For example, Figure $8 C$ shows a linear regression between the firing rates of the SIRT against the SCT for the cell depicted in Figure $2 A-C$. The resulting slope was close to unity (1.1); however, the constant was $5.8 \mathrm{~Hz}$, suggesting that the response across duration was similar in the two tasks but scaled with larger responses in the SCT. When the regressions were performed between the same tasks but different modality (SCT-auditory vs SCT-visual and SIRT-auditory vs SIRT-visual), the slope distribution for the interval-selective cells was around one (Fig. $8 D$ ) and the constant distribution was centered on zero (Fig. $8 F$ ). These results support the notion that interval tuning was not strongly affected by the modality used to drive the temporal behavior. In contrast, the 
regressions performed on the cell activity between same modality but different task (SIRT-auditory vs SCT-auditory and SIRT-visual vs SCT-visual), showed a slope distribution for the intervalselective cells that peaked at 1 but was skewed for larger values (Fig. 8E). These results suggest that the cells showed either a similar response profile between SCT and SIRT (slopes close to 1) or a rate of change in activity among durations that was larger for SCT than SIRT (slopes larger than 1). This last result provides evidence for tuning sharpening during the SCT for a subpopulation of MPC cells.

The fact that a large population of interval-tuned neurons showed similar preferred intervals and response profiles for the tested durations across the four task combination provides support to the hypothesis that MPC is part of a core timing mechanism that is engaged in multiple timing behaviors.

\section{A gain mechanism to encode the number of produced intervals in a rhythmic sequence}

The cells depicted in Figures $2 A-C$ and $7 A-E$ show another property of interval tuning in MPC, namely that although the preferred intervals of these cells were similar across tasks, the discharge rate at the preferred duration was larger in the SCT than the SIRT. Accordingly, at the population level we observed a significantly larger maximum discharge rate for cells with similar preferred intervals $(<100 \mathrm{~ms}$ in PI difference) during the SCT with respect to SIRT, for both the visual and auditory conditions (Fig. 8B, MannWhitney $U$ tests; Table 6). In addition, the constant of the linear regressions between same modality but different task (SIRTauditory vs SCT-auditory and SIRTvisual vs SCT-visual), was shifted to the right (Fig. 8G). Hence, a large population of cells showed positive values in the constant or intercept of the regression, suggesting a coordinated increase in discharge rate for the SCT with respect of SIRT, as depicted in Figure $8 C$. These findings suggest the existence of a gain mechanism as the possible neural representation for the total number of produced intervals in a sequence, where the discharge rate of the tuned cells increases between one and six produced intervals, although the preferred intervals are similar across conditions.

\section{Discussion}

The present study describes the following functional properties in MPC cells: (1) cells in MPC are tuned to duration in different tapping tasks; (2) there was interval-tuning invariance across tasks (SCT and SIRT) and modalities in a large population of neurons; (3) during the SCT, a large cell population was tuned to both the sequential and temporal structure of the task; and (4)
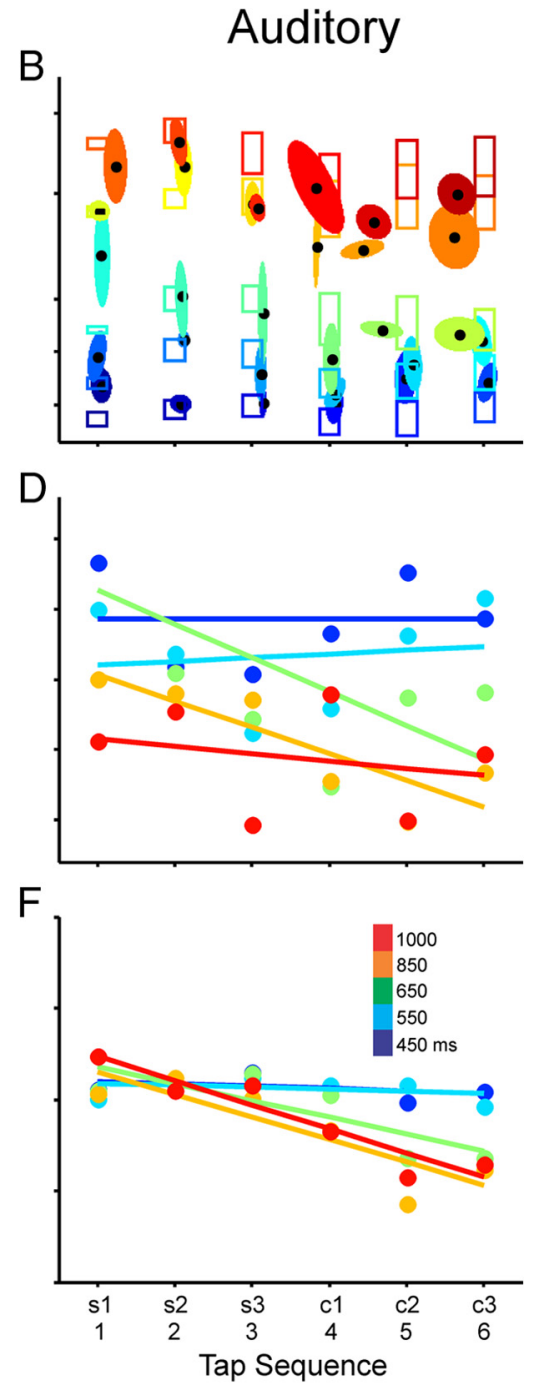

Figure 6. Decoding the sequential and temporal structure of the SCT. $\boldsymbol{A}, \boldsymbol{B}$, Bivariate normal distributions of the Bayesian decoded values for produced duration/ordinal sequence using the activity of double-tuned cell populations recorded during simila is proportional to the total SD of the decoded values. The bottom and top of the color boxes correspond to the mean \pm SD of the produced intervals on the trials used for decoding. The gradient in the color code changes as a function of sequence order and target ine decoding ellipses and the behavior boxes. C, D, Bias between the decoded and produced durations as a function of the tapping sequence across the five target intervals, using the color code shown in the inset in $\boldsymbol{F}$. $\boldsymbol{E}, \boldsymbol{F}$, Bias between the decoded and the actual sequential order as a function of the tapping sequence, across the five target intervals. The results for the visual (left) and auditory (right) marker conditions are shown.

there is a possible gain mechanism for encoding the number of intervals in a sequence of temporalized movements. We discuss these results separately below.

This study gives, as far as we know, the first empirical evidence for duration tuning in the behaving monkey. The existence of interval tuning was inferred from learning and generalization studies of time intervals (Nagarajan et al., 1998; Meegan et al., 2000; Bartolo and Merchant, 2009; Heron et al., 2012) and has been suggested in conceptual papers (Ivry, 1996). These results suggest that the MPC contains a representation of interval, where different populations of interval-tuned cells are activated depending on the duration of the produced interval. Cell tuning is an encoding mechanism widely used in cortical cells to represent sensory, cognitive, and motor information (deCharms and Zador, 2000; Merchant et al., 2012). Studies of tuning have demonstrated that most cortical neurons carry information about a 


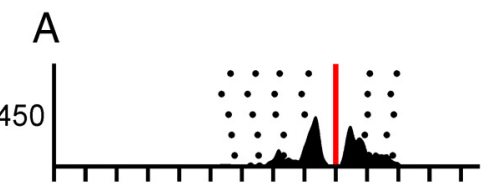

B
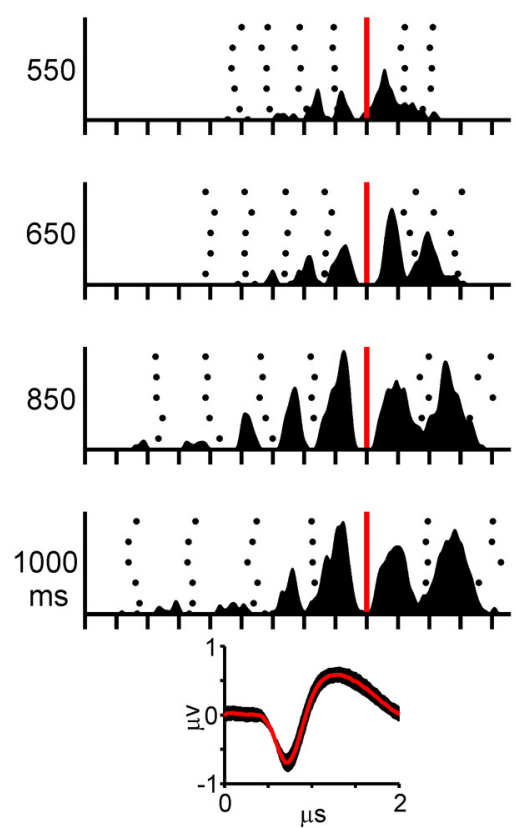

$E$

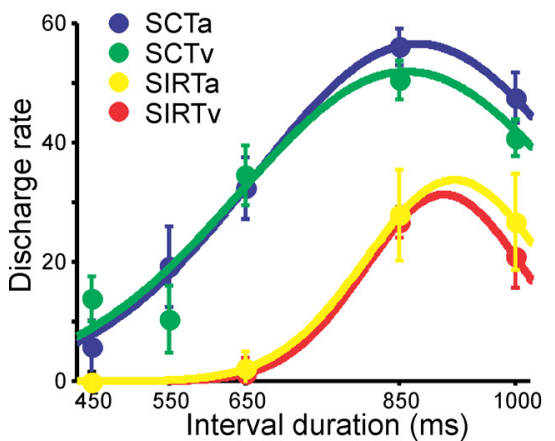

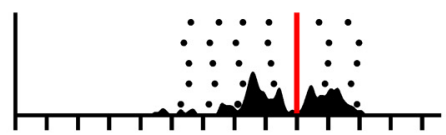
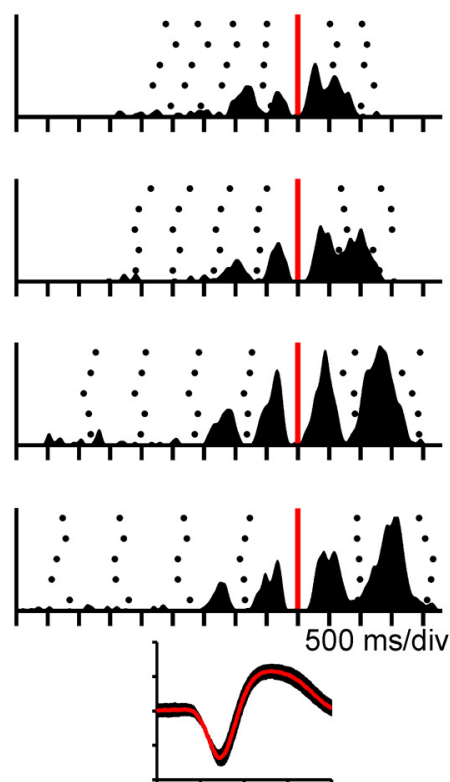

$\mathrm{F}$

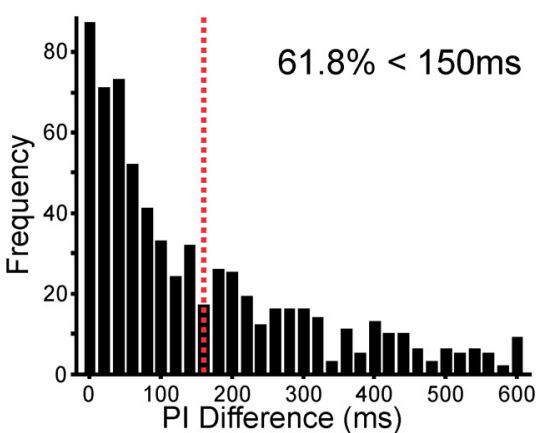

C
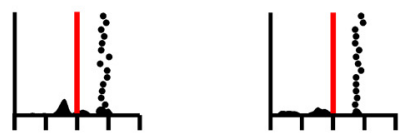
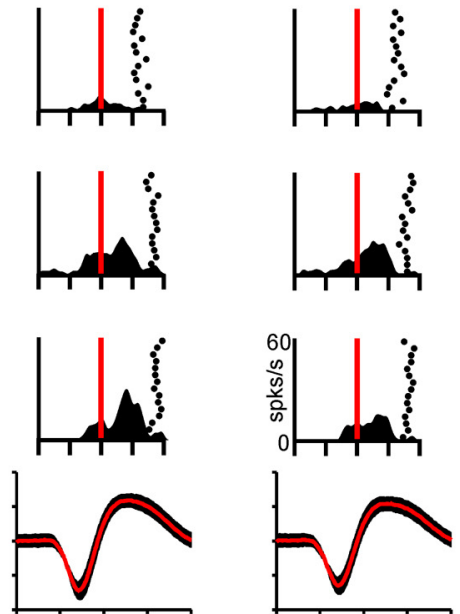

G

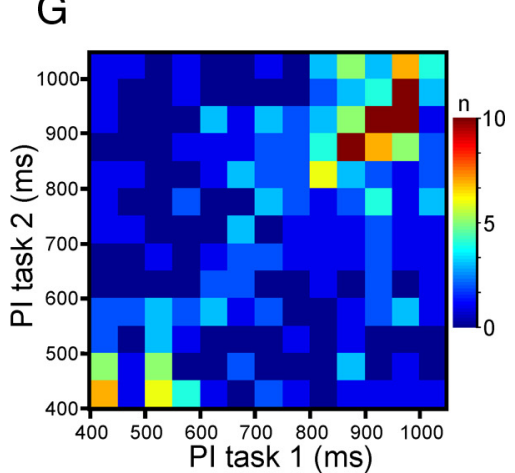

Figure 7. Interval tuning across tasks and sensory modalities. $\boldsymbol{A}-\boldsymbol{D}$, Average spike-density functions of the responses of an interval-tuned cell with a long preferred interval across different temporal contexts. The mean (red curve) and SD (black area) of the action potential waveform during each task combination is shown at the bottom. $A$, SCT in the auditory condition. $B$, SCT in the visual condition. C, SIRT in the auditory condition. D, SIRT in the visual condition. In $\boldsymbol{A}$ and $\boldsymbol{B}$, the raster histograms are aligned (red line) to the first tap of the continuation phase, whereas in $\boldsymbol{C}$ and $\boldsymbol{D}$, they are aligned to the first tapping movement. $\boldsymbol{E}$, Tuning functions for the cell in $\boldsymbol{A}-\boldsymbol{D}$, where the mean \pm SEM of the discharge rate is plotted as a function of the target interval. The continuous lines correspond to the significant Gaussian fits. SCTa, Auditory SCT (blue); SCTv, visual SCT (green); SIRTa, auditory SIRT (yellow); SIRTv, visual SIRT (red). F, Distribution of the PI difference for the cells tuned during different task pairs. The Dotted red line corresponds to the $150 \mathrm{~ms}$ PI difference. G, Density plot of the Pls for the same cells between all possible combinations of task pairs (task 1 vs task 2). The color code represents the number ( $\mathrm{n}$ ) of cells with different PI combinations between tasks.

Table 3. Statistics of the distributions of preferred interval differences between the specified number of tasks

\begin{tabular}{lllc}
\hline & Two & Three & Four \\
\hline Number & 272 & 100 & 16 \\
Median & 120.2 & 127.2 & 74.3 \\
Mean & 173.2 & 173.1 & 120.2 \\
SD & 164.1 & 161.4 & 126.8 \\
$\%<150 \mathrm{~ms}$ & 57.7 & 56 & 69.8 \\
\hline
\end{tabular}

limited range of values or a particular behavioral feature, with an orderly decrease in discharge rate around a preferred value (Merchant et al., 2012). Thus, interval timing, as in the case of numerosity and spatial magnitudes (Georgopoulos et al., 1986; Walsh, 2003; Buhusi and Meck, 2005; Nieder et al., 2006), is represented as an abstract quantity in MPC by a population of interval-tuned cells. The distribution of preferred intervals was skewed for intervals around $850 \mathrm{~ms}$ in the four task combinations, which could reflect that monkeys have a behavioral timing repertoire where this interval is prevalent. Indeed, we found that the constant error for this interval was close to zero across tasks. Additional experiments are needed to determine whether tuning to interval is an emergent property of MPC cells that depends on the local integration of graded inputs or is a distributed representation of time throughout the CBGT (Matell and Meck, 2004).

Importantly, the present findings indicate that a large population of tuned cells in MPC showed similar preferred intervals across tapping behaviors that varied in the number of produced intervals and the modality used to drive temporal processing. 
A

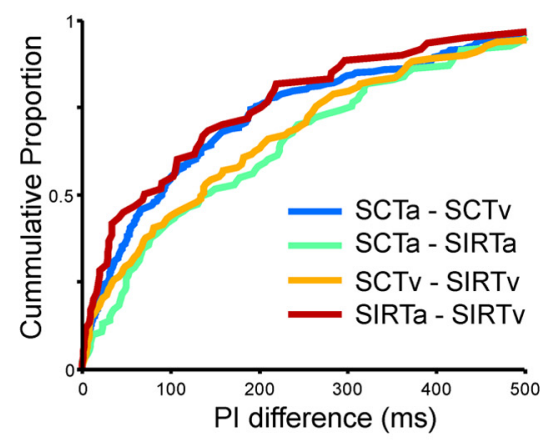

B

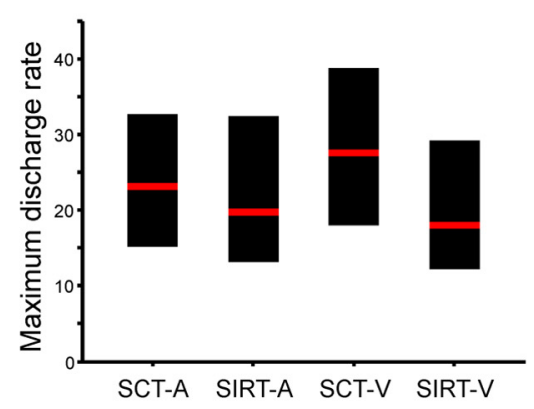

D

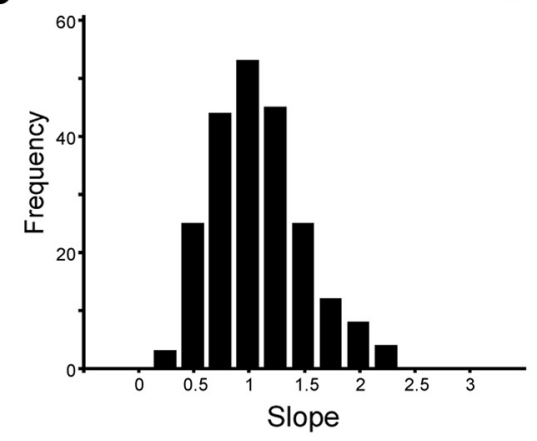

E

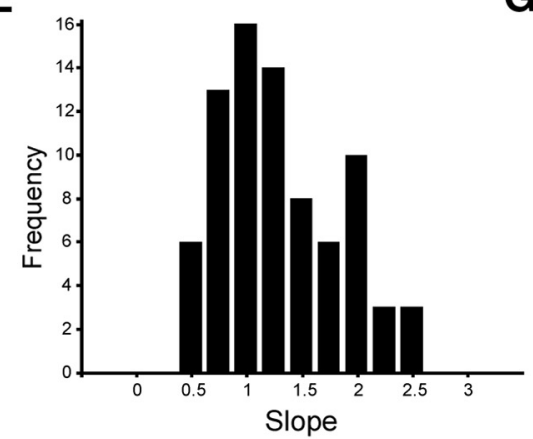

F

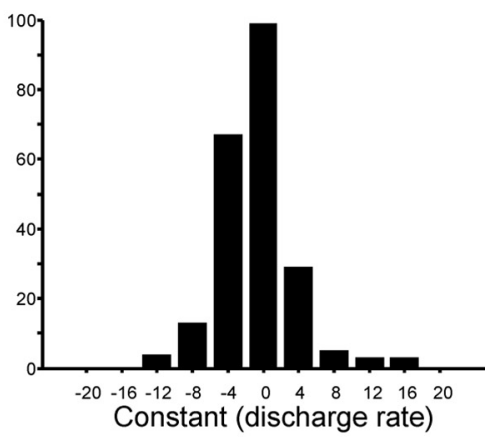

G

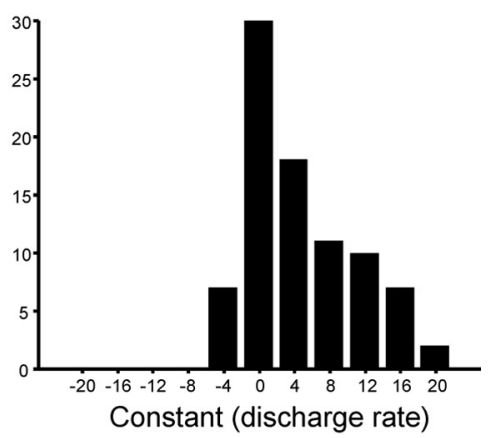

C

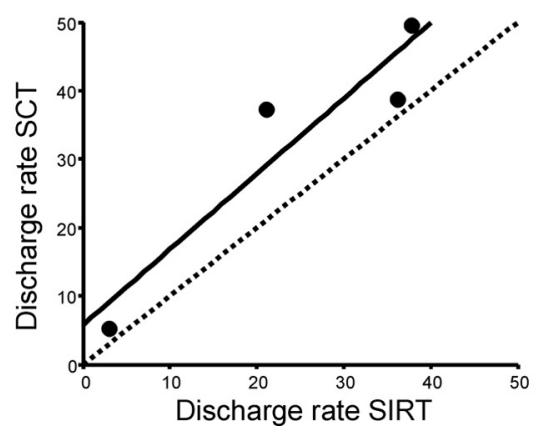

Figure 8. Gain mechanism for the number of produced intervals. $A$, Cumulative distributions for the PI difference for tuned cells between specific task pairs. The color code for task pairs is given in the inset. $\boldsymbol{B}$, Interquartile ranges (black bars) and medians (red line within bars) of the maximum discharge rate of cells across the four task combinations. Only cells that were interval tuned in at least two tasks and showed a small difference $(<100 \mathrm{~ms})$ in their preferred interval across tasks were included in the analysis. $C$, Discharge rates of the SIRT against the SCT of the cell in Figure $2 A-C$, where the dotted line is the diagonal and the black line is the predicted function of the corresponding linear regression. $D, E$, Histograms of the slope and constant of the significant linear regression models for single-cell discharge rates between same tasks but different modality [SCTa vs SCTv $(n=200)$ and SIRTa vs SIRTv $(n=33)$ ]. $F, G$, Histograms of the slope and constant of the significant linear regression models for single-cell discharge rates between same modality but different task regressions [SIRTa vs SCTa $(n=49)$ and SIRTv vs SCTv ( $n=61)$ ]. SCTa, Auditory SCT; SCTv, visual SCT; SIRTa, auditory SIRT; SIRTv, visual SIRT.

Table 4. Statistics of the distributions of preferred interval differences between the specified tasks pairs

\begin{tabular}{lcccl}
\hline & SIRTa-SIRTv & SCTa-SCTv & SCTa-SIRTa & SCTv-SIRTV \\
\hline Number & 60 & 218 & 93 & 109 \\
Median & 78.7 & 89.2 & 143.5 & 136.8 \\
Mean & 132.7 & 143.1 & 187.5 & 178.1 \\
SD & 152.8 & 151.6 & 159.6 & 168.4 \\
$\%<150 \mathrm{~ms}$ & 68.3 & 66 & 51.6 & 54.1 \\
\hline
\end{tabular}

SCTa, SCT auditory; SCTv, SCT visual; SIRTa, SIRT auditory; SIRTV, SIRT visual.

Hence, interval-tuning invariance across the four tested task combinations suggests that the medial premotor areas tag the timed durations as a context-independent neural signal. Tuning invariance to low-order parameters also has been observed in the upper hierarchies of processing in the visual and auditory sys-
Table 5. $p$ values of the Kolmogorov-Smirnov tests between the distributions of preferred interval differences between the specified tasks pairs

\begin{tabular}{llll}
\hline & SCTa-SIRTa & SCTv-SIRTv & SIRTa-SIRTV \\
\hline SCTa-SCTv & 0.007 & 0.066 & 0.423 \\
SCTa-SIRTa & & 0.471 & 0.007 \\
SCTv-SIRTv & & & 0.058 \\
\hline
\end{tabular}

SCTa, SCT auditory; SCTV, SCT visual; SIRTa, SIRT auditory; SIRTv, SIRT visual.

Table 6. $p$ values of the Mann-Whitney $U$ tests between the intervals with the maximum discharge rates for the specified tasks

\begin{tabular}{lll}
\hline & SCTv & SIRTa \\
\hline SCTa & 0.08 & 0.03 \\
SIRTV & 0.0000001 & 0.201
\end{tabular}

SCTa, SCT auditory; SCTv, SCT visual; SIRTa, SIRT auditory; SIRTV, SIRT visual. 
tems, where there are cells that show selective responses to abstract features of complex stimuli (Sadagopan and Wang, 2008). For example, cells in the inferotemporal cortex show visual object-selective cells that exhibit tuning invariance to complex shapes (such as faces) submitted to translation, scale changes, and a limited rotation in depth (Logothetis and Sheinberg, 1996) and probably are associated with the perceptual recognition of objects. The interval-tuning invariance observed here supports the notion that the CNS needs a reliable representation of duration in different behavioral circumstances. Therefore, these findings are consistent with the existence of a core timing mechanism that is engaged in multiple timing behaviors and that includes the MPC (Merchant et al., 2008a, 2013; Harrington et al., 2010).

On the other hand, the ability to capture and interpret the beats in a rhythmic pattern allows people to move and dance in time to music (Phillips-Silver and Trainor, 2005). Thus, rhythmic behaviors depend on intricate loops of perception and action, where temporal processing can be engaged during the synchronization of movements with sensory information or during the internal generation of movement sequences (Janata and Grafton, 2003). The SCT has been an instrumental paradigm to study these processes, and a recent neurophysiological study has shown that sensorimotor loops engaged during this task may depend on the cyclic interplay between different neuronal chronometers in MPC that quantify in their instantaneous activity the time passed and the remaining time for an action (Merchant et al., 2011). The present findings suggest that MPC also uses interval tuning as an abstract representation of the passage of time. Interestingly, interval tuning during the SCT is multiplexed with the tuning to ordinal sequence in the same population of cells. Therefore, the brain might use a cell population signal that works as the notes of a musical score to represent both the duration of the produced interval and the rank order of the interval that is executed in the learned SCT sequence. Hence, not only is MPC involved in the independent encoding of the multiple movement sequences (Tanji and Shima, 1994; Shima and Tanji, 1998) or the timing of isolated movements (Mita et al., 2009) but there is also a two-dimensional representation of sequence order and timing in populations of MPC cells during rhythmic behaviors such as the SCT. It is important to mention that previous studies have shown that MPC neurons modulate their activity according to the number of remaining movements in a sequence to receive a reward, suggesting that they might encode the timing of reward (Sohn and Lee, 2007). Although it is possible that some of the sequence order signals reported here could be related to the time remaining to reward, the distribution of preferred ordinal sequence in the population is not biased toward the last elements of the sequence, particularly for the auditory marker condition (Fig. $4 E$ ), supporting the notion that the double-tuned cells were multiplexing ordinal and temporal structure of the SCT. Finally, the multiplexing of ordinal sequence and duration also may be a neural signal shared throughout the CBGT, since previous studies have described neural responses associated with interval duration and the organization of sequential motor behavior in the neostriatum (Kermadi and Joseph, 1995; Miyachi et al., 1997; Matell et al., 2003a,b; Jin et al., 2009; Bartolo et al., 2012).

Time-related cell responses have been reported in parietal (Leon and Shadlen, 2003) and prefrontal (Brody et al., 2003; Genovesio et al., 2009) cortex, putamen (Jin et al., 2009), thalamus (Tanaka, 2007), MPC (Mita et al., 2009; Merchant et al., 2011), and motor cortex (Lebedev et al., 2008). Hence, in agreement with the neuroimaging literature, these neurophysiological studies point to a highly distributed processing of temporal information by CBGT networks (Meck et al., 2008). Most of these studies report ramping activity during a variety of timing tasks. A fundamental characteristic of climbing activity is its instantaneous nature and the fact that it normally peaks at the time of an anticipated motor response, suggesting its involvement in the temporal construction of motor intentions and actions (Merchant et al., 2004; Maimon and Assad, 2006; Renoult et al., 2006). In contrast, the cells that are tuned to an interval may represent different aspects of temporal processing that can be dissociated from the motor response.

As a final point, the present results suggest the existence of a gain mechanism for encoding the total number of produced intervals in a sequence, where larger numbers of produced intervals were associated with a multiplicative response scaling across durations and the corresponding increase in discharge rate in the preferred interval of tuned cells. Psychophysical studies in both perceptual and production tasks have demonstrated that temporal variability decreases as a function of the number of intervals to be timed (Keele et al., 1989; Ivry and Hazeltine, 1995; Merchant et al., 2008a,b). Hence, the decrease in variability with the number of timed intervals could be the result of the increase in discharge rate in the preferred interval of MPC cells. Gain mechanisms have been described for the combination of retinal and gaze signals (Andersen et al., 1985) and for the representation of states of visual attention (Treue and Martinez Trujillo, 1999) and have been regarded as a major computational principle of nonlinear neuronal processing (Salinas and Thier, 2000). The MPC may use this gain principle to represent the total number of produced intervals in a sequence, while through tuning it can multiplex interval and ordinal sequence in a rhythmic paradigm. Needless to say, studies using different total numbers of intervals, instead of just one (SIRT) and six (SCT), are necessary to confirm this hypothesis.

\section{References}

Andersen RA, Essick GK, Siegel RM (1985) Encoding of spatial location by posterior parietal neurons. Science 230:456-458. CrossRef Medline

Bartolo R, Merchant H (2009) Learning and generalization of time production in humans: rules of transfer across modalities and interval durations. Exp Brain Res 197:91-100. CrossRef Medline

Bartolo R, Prado L, Merchant H (2012) The oscillatory activity of the primate putamen is associated with the temporal and sequential structure of a synchronization-continuation tapping task. Paper presented at $42 \mathrm{nd}$ Congress of the Society for Neuroscience, New Orleans, LA, October.

Brody CD, Hernández A, Zainos A, Romo R (2003) Timing and neural encoding of somatosensory parametric working memory in macaque prefrontal cortex. Cereb Cortex 13:1196-1207. CrossRef Medline

Bueti D, Walsh V, Frith C, Rees G (2008) Different brain circuits underlie motor and perceptual representations of temporal intervals. J Cogn Neurosci 20:204-214. CrossRef Medline

Buhusi CV, Meck WH (2005) What makes us tick? Functional and neural mechanisms of interval timing. Nat Rev Neurosci 6:755-765. CrossRef Medline

Buonomano DV, Laje R (2010) Population clocks: motor timing with neural dynamics. Trends Cogn Sci 14:520-527. CrossRef Medline

Coull JT, Cheng RK, Meck WH (2011) Neuroanatomical and neurochemical substrates of timing. Neuropsychopharmacology 36:3-25. CrossRef Medline

Dayan P, Abbott LF (2001) Theoretical neuroscience, computational and mathematical modeling of neural systems. London: MIT.

deCharms RC, Zador A (2000) Neural representation and the cortical code. Annu Rev Neurosci 23:613-647. CrossRef Medline

Dickey AS, Suminski A, Amit Y, Hatsopoulos NG (2009) Single-unit stability using chronically implanted multielectrode arrays. J Neurophysiol 102:1331-1339. CrossRef Medline 
Diehl RL, Lotto AJ, Holt LL (2004) Speech perception. Annu Rev Psychol 55:149-179. CrossRef Medline

Draper NR, Smith H (1981) Applied regression analysis. New York: Wiley. Genovesio A, Tsujimoto S, Wise SP (2009) Feature- and order-based timing representations in the frontal cortex. Neuron 63:254-266. CrossRef Medline

Georgopoulos AP, Schwartz AB, Kettner RE (1986) Neuronal population coding of movement direction. Science 233:1416-1419. CrossRef Medline

Gibbon J, Malapani C, Dale CL, Gallistel C (1997) Toward a neurobiology of temporal cognition: advances and challenges. Curr Opin Neurobiol 7:170-184. CrossRef Medline

Hanes DP, Thompson KG, Schall JD (1995) Relationship of presaccadic activity in frontal eye field and supplementary eye field to saccade initiation in macaque: Poisson spike train analysis. Exp Brain Res 103:85-96. Medline

Harrington DL, Zimbelman JL, Hinton SC, Rao SM (2010) Neural modulation of temporal encoding, maintenance, and decision processes. Cereb Cortex 20:1274-1285. CrossRef Medline

Hastie T, Tibshirani R, Friedman J (2001) The elements of statistical learning: data mining, inference, and prediction. New York: Springer.

Heron J, Aaen-Stockdale C, Hotchkiss J, Roach NW, McGraw PV, Whitaker D (2012) Duration channels mediate human time perception. Proc Biol Sci 279:690-698. CrossRef Medline

Ivry RB (1996) The representation of temporal information in perception and motor control. Curr Opin Neurobiol 6:851-857. CrossRef Medline

Ivry RB, Hazeltine RE (1995) Perception and production of temporal intervals across a range of durations: evidence of a common timing mechanism. J Exp Psychol Hum Perform 21:3-18. CrossRef Medline

Janata P, Grafton ST (2003) Swinging in the brain: shared neural substrates for behaviors related to sequencing and music. Nat Neurosci 6:682-687. CrossRef Medline

Jin DZ, Fujii N, Graybiel AM (2009) Neural representation of time in cortico-basal ganglia circuits. Proc Natl Acad Sci U S A 106:19156-19161. CrossRef Medline

Johnson RA, Wichern DW (1998) Applied multivariate statistical analysis. Upper Saddle River, NJ: Prentice Hall.

Karmarkar UR, Buonomano DV (2007) Timing in the absence of clocks: encoding time in neural network states. Neuron 53:427-438. CrossRef Medline

Keele S, Nicoletti R, Ivry R, Pokorny R (1989) Mechanisms of perceptual timing: beat-based or interval-based judgments? Psychol Res 50:251-256. CrossRef

Kermadi I, Joseph JP (1995) Activity in the caudate nucleus of monkey during spatial sequencing. J Neurophysiol 74:911-933. Medline

Lebedev MA, O’Doherty JE, Nicolelis MA (2008) Decoding of temporal intervals from cortical ensemble activity. J Neurophysiol 99:166-186. CrossRef Medline

Leon MI, Shadlen MN (2003) Representation of time by neurons in the posterior parietal cortex of the macaque. Neuron 38:317-327. CrossRef Medline

Logothetis NK, Sheinberg DL (1996) Visual object recognition. Annu Rev Neurosci 19:577-621. CrossRef Medline

Macar F, Coull J, Vidal F (2006) The supplementary motor area in motor and perceptual time processing: fMRI studies. Cogn Process 7:89-94. CrossRef Medline

Maimon G, Assad JA (2006) A cognitive signal for the proactive timing of action in macaque LIP. Nat Neurosci 9:948-955. CrossRef Medline

Matell MS, Meck WH (2004) Cortico-striatal circuits and interval timing: coincidence detection of oscillatory processes. Brain Res Cogn Brain Res 21:139-170. CrossRef Medline

Matell MS, Meck WH, Nicolelis MA (2003a) Interval timing and the encoding of signal duration by ensembles of cortical and striatal neurons. Behav Neurosci 117:760-773. CrossRef Medline

Matell MS, Meck WH, Nicolelis MAL (2003b) Integration of behavior and timing: anatomically separate systems or distributed processing? In: Functional and neural mechanisms of interval timing (Meck WH, ed), pp 371-392. Boca Raton, FL: CRC.

Meck WH, Penney TB, Pouthas V (2008) Cortico-striatal representation of time in animals and humans. Curr Opin Neurobiol 18:145-152. CrossRef Medline
Meegan DV, Aslin RN, Jacobs RA (2000) Motor timing learned without motor training. Nat Neurosci 3:860-862. CrossRef Medline

Merchant H, Georgopoulos AP (2006) Neurophysiology of perceptual and motor aspects of interception. J Neurophysiol 95:1-13. Medline

Merchant H, Perez O (2009) Neurophysiology of interceptive behavior in the primate: encoding and decoding target parameters in the parietofrontal system. In: Coherent behavior in neural networks (Josic K, Matias M, Romo R, Rubin J, eds), Springer Series in Computational Neuroscience, Vol 3, pp 191-206. New York: Springer.

Merchant H, Battaglia-Mayer A, Georgopoulos AP (2004) Neural responses during interception of real and apparent circularly moving stimuli in motor cortex and Area 7a. Cereb Cortex 14:314-331. CrossRef Medline

Merchant H, Zarco W, Prado L (2008a) Do we have a common mechanism for measuring time in the hundreds of millisecond range? Evidence from multiple-interval timing tasks. J Neurophysiol 99:939-949. CrossRef Medline

Merchant H, Zarco W, Bartolo R, Prado L (2008b) The context of temporal processing is represented in the multidimensional relationships between timing tasks. PloS One 3:e3169:1-9. CrossRef Medline

Merchant H, Naselaris T, Georgopoulos AP (2008c) Dynamic sculpting of directional tuning in the primate motor cortex during $3 \mathrm{D}$ reaching. J Neurosci 28:9164-9172. CrossRef Medline

Merchant H, Zarco W, PérezO, Prado L, Bartolo R (2011) Measuring time with different neural chronometers during a synchronization-continuation task. Proc Natl Acad Sci U S A 108:19784-19789. CrossRef Medline

Merchant H, de Lafuente V, Peña-Ortega F, Larriva-Sahd J (2012) Functional impact of interneuronal inhibition in the cerebral cortex of behaving animals. Prog Neurobiol 99:163-178. CrossRef Medline

Merchant H, Harrington D, Meck WH (2013) Neural basis of the perception and estimation of time. Annu Rev Neurosci, in press.

Mita A, Mushiake H, Shima K, Matsuzaka Y, Tanji J (2009) Interval time coding by neurons in the presupplementary and supplementary motor areas. Nat Neurosci 12:502-507. CrossRef Medline

Miyachi S, Hikosaka O, Miyashita K, Kárádi Z, Rand MK (1997) Differential roles of monkey striatum in learning of sequential hand movement. Exp Brain Res 115:1-5. CrossRef Medline

Nagarajan SS, Blake DT, Wright BA, Byl N, Merzenich MM (1998) Practicerelated improvements in somatosensory interval discrimination are temporally specific but generalize across skin location, hemisphere, and modality. J Neurosci 18:1559-1570. Medline

Nieder A, Diester I, Tudusciuc O (2006) Temporal and spatial enumeration processes in the primate parietal cortex. Science 313:1431-1435. CrossRef Medline

Perez O, Kass RE, Merchant H (2013) Trial time warping to discriminate stimulus-related from movement-related neural activity. J Neurosci Methods 212:203-210. CrossRef Medline

Phillips-Silver J, Trainor LJ (2005) Feeling the beat: movement influences infant rhythm perception. Science 308:1430. CrossRef Medline

Rakitin BC, Gibbon J, Penney TB, Malapani C, Hinton SC, Meck WH (1998) Scalar expectancy theory and peak-interval timing in humans. J Exp Psychol Anim Behav Process 24:15-33. CrossRef Medline

Renoult L, Roux S, Riehle A (2006) Time is a rubberband: neuronal activity in monkey motor cortex in relation to time estimation. Eur J Neurosci 23:3098-3108. CrossRef Medline

Sadagopan S, Wang X (2008) Level invariant representation of sounds by populations of neurons in primary auditory cortex. J Neurosci 28:34153426. CrossRef Medline

Salinas E, Thier P (2000) Gain modulation: a major computational principle of the central nervous system. Neuron 27:15-21. CrossRef Medline

Shima K, Tanji J (1998) Both supplementary and presupplementary motor areas are crucial for the temporal organization of multiple movements. J Neurophysiol 80:3247-3260. Medline

Sohn JW, Lee D (2007) Order-dependent modulation of directional signals in the supplementary and presupplementary motor areas. J Neurosci 27: 13655-13666. CrossRef Medline

Tanaka M (2007) Cognitive signals in the primate motor thalamus predict saccade timing. J Neurosci 27:12109-12118. CrossRef Medline

Tanji J (2001) Sequential organization of multiple movements: involvement of cortical motor areas. Annu Rev Neurosci 24:631-651. CrossRef Medline

Tanji J, Shima K (1994) Role for supplementary motor area cells in plan- 
ning several movements ahead. Nature 371:413-416. CrossRef Medline

Treue S, Martínez Trujillo JC (1999) Feature-based attention influences motion processing gain in macaque visual cortex. Nature 399:575-579. CrossRef Medline

Walsh V (2003) A theory of magnitude: common cortical metrics of time, space and quantity. Trends Cogn Sci 7:483-488. CrossRef Medline
Wiener M, Turkeltaub P, Coslett HB (2010) The image of time: a voxel-wise meta-analysis. Neuroimage 49:1728-1740. CrossRef Medline

Zarco W, Merchant H, Prado L, Mendez JC (2009) Subsecond timing in primates: comparison of interval production between human subjects and rhesus monkeys. J Neurophysiol 102:3191-3202. CrossRef Medline 